ESAIM: M2AN 47 (2013) 1185-1205

DOI: $10.1051 / \mathrm{m} 2 \mathrm{an} / 2012064$
ESAIM: Mathematical Modelling and Numerical Analysis

www.esaim-m2an.org

\title{
QUADRATIC FINITE ELEMENTS WITH NON-MATCHING GRIDS FOR THE UNILATERAL BOUNDARY CONTACT
}

\author{
S. Auliac ${ }^{1}$, Z. Belhachmi ${ }^{2}$, F. Ben Belgacem ${ }^{3,4}$ And F. Hecht ${ }^{5}$
}

\begin{abstract}
We analyze a numerical model for the Signorini unilateral contact, based on the mortar method, in the quadratic finite element context. The mortar frame enables one to use non-matching grids and brings facilities in the mesh generation of different components of a complex system. The convergence rates we state here are similar to those already obtained for the Signorini problem when discretized on conforming meshes. The matching for the unilateral contact driven by mortars preserves then the proper accuracy of the quadratic finite elements. This approach has already been used and proved to be reliable for the unilateral contact problems even for large deformations. We provide however some numerical examples to support the theoretical predictions.
\end{abstract}

Mathematics Subject Classification. 35J85, 65N30, 74M15.

Received May 11, 2012. Revised September 24, 2012.

Published online June 17, 2013.

\section{INTRODUCTION}

High-order finite elements are more and more relevant for complex systems since they allow for large computations with increased accuracy. Engineers of unilateral contact may be interested in as an afforded response to capture small structures in their numerical simulations. Letting apart the linear finite element to which a wide literature is devoted. We refer to the books $[22,31,44]$ as classical references, then to [12] for earlier a priori error estimates and to $[24,29,35]$ for more recent studies which improve former results in the literature. Conducting the convergence analysis of the finite element approximation applied to unilateral problems turns out to be a pain in the neck, presumably because they are expressed by variational inequalities. Unlike the linear variational equations for which a general theory of the finite element methods has been developed, specific tools should be used for the unilateral contact inequality according to the degree of the finite elements. Here, we are interested in the Signorini boundary contact. Some of the mixed finite elements that are performing tools to efficiently handle the numerical locking caused by the nearly incompressible materials in elasticity, are based on quadratic elements (see [11]). One may think for example of Taylor-Hood elements already used for the unilateral contact $($ see $[6,28,40])$. These all are as many reasons why it is worth to study quadratic finite elements for the unilateral

Keywords and phrases. Unilateral contact conditions, quadratic finite elements, non-matching grids, mortar matching.

1 LJLL, C.N.R.S. Université Pierre et Marie Curie, B.C. 187, 4 place Jussieu, 75252 Paris Cedex 05, France.

auliac@ann.jussieu.fr

2 LMIA, EA CNRS, Université deq Haute Alsace, Rue des Frères Lumière, 68096 Mulhouse, France.

zakaria.belhachmi@uha.fr

3 LMAC, EA 2222, Université de Technologie de Compiègne, BP 20529, 60205 Compiègne Cedex, France.

faker.ben-belgacem@utc.fr

4 I2M (UMR CNRS 5295), Site ENSCBP, 16 Avenue Pey Berland, 33607 Pessac Cedex, France.

5 LJLL, C.N.R.S. Université Pierre et Marie Curie, B.C. 187, 4 place Jussieu, 75252 Paris Cedex 05, France. hecht@ann.jussieu.fr 
contact. We are involved in non-matching meshes and in the use of the mortar method. Deep attention has been successfully devoted to a single mesh context in Signorini's problem or in the case of compatible grids that is the meshes within each component of the system to handle numerically coincide at the interfaces. Detailed studies with optimal convergence estimates are available for those finite element methods with matching grids (see [4]). When the meshes are constructed independently, resulting in dissimilar grids that do not match at interfaces, a substantial implementation and validation work have been achieved (see $[20,21,30,34,37,38]$ ) either in unilateral or bilateral contact with or without friction. We recommend in particular the interesting survey provided in [33]. In the numerical analysis chapter, only few attempts have been realized for the unilateral configurations (see $[27,30]$ ). We pursue here a study of some numerical modelings of unilateral contact in the frame of the mortar-matching for finite elements with degree two, applied to the primal variational Signorini Inequality. A literature exists on the linear mortar finite element method for the Signorini problem. We refer to $[3,5,25,26,42]$ without being exhaustive. Users are recommended not to enforce the unilateral conditions on the discrete solution before running the mortar projection. Such a mortar matching, first introduced in [8], allows to express the unilateral inequalities, in the discrete level, on traces built on the same grid and avoids by then possible instabilities along interfaces. It is nowadays widely known that writing unilateral inequalities directly on the approximated solution at the interfaces as done in the classical node-to-segment treatments, generates low numerical accuracy. We refer to $[21,32,43]$ for a worthy discussion about the efficiency of the mortar method in interface contact mechanics.

Our willing is to focus only on the mortaring and technical issues related to, for conciseness. We exclude therefore any other features which are doubtless as important in unilateral contact and that are either already successfully handled or under consideration in many research teams. We pay all our attention to the mortar projections along interfaces of dissimilar grids, to the way the unilateral contact is enforced at the discrete level and finally to show how the combination of both yields optimal convergence results, owing to Falk's Lemma. The outline of the paper is as follows. Section 2 is a presentation of some models where unilateral contact is involved. We describe briefly the static problem of the unilateral contact between two elastic bodies, the solution to be computed is the displacement field. We recall also the unilateral conditions in the case of the Laplace equation. The regularity issue for the solution of the Signorini-Laplace problem is discussed at the end of the section. In Section 3, we introduce the mortar framework for the quadratic finite elements and the unilateral conditions are then expressed at the nodes of the interfaces. After recalling a suitable version of Falk's Lemma, we dedicate Section 4 to the numerical analysis of the mortar quadratic finite element method for the SignoriniLaplace problem. The convergence rates we exhibit in our main result, Theorem 4.1, are similar to the case where matching grids are used. As a result, using incompatible grids in the simulation of unilateral contact does not degrade the accuracy when mortar-matching drives the communication between different meshes. The final section is dedicated to some numerical experiences to check the theoretical predictions. Lastly, in Section 5, we describe and comment some experiences realized in Freefem++ to assess the theoretical predictions proved here. Some notations. Let $\mathscr{C}(\Omega)$ be the space of real valued continuous function on a given domain $\Omega$. The Lebesgue space of square integrable functions $L^{2}(\Omega)$ is endowed with the natural inner product $(\cdot, \cdot)_{L^{2}(\Omega)}$; the associated norm is $\|\cdot\|_{L^{2}(\Omega)}$. The Sobolev space $H^{1}(\Omega)$ involves all the functions that are in $L^{2}(\Omega)$ so as their first order derivatives. It is provided with the norm $\|\cdot\|_{H^{1}(\Omega)}$ and the semi-norm is denoted by $|\cdot|_{H^{1}(\Omega)}$. For any portion of the boundary $\Upsilon \subset \partial \Omega$, the space $H_{0}^{1}(\Omega, \Upsilon)$ contains all the functions of $H^{1}(\Omega)$ that vanish on $\Upsilon$. We recall that, for any $\theta \in] 0,1\left[\right.$, the Sobolev space $H^{\theta}(\Upsilon)$ can be obtained by a Hilbertian interpolation between $H^{1}(\Upsilon)$ and $L^{2}(\Upsilon)$ and $H^{-\theta}(\Upsilon)$ is the dual space $\left(H^{\theta}(\Upsilon)\right)^{\prime}$. Notice finally that any function $v \in H_{0}^{1}(\Omega, \partial \Omega \backslash \Upsilon)$ whose Laplacian is in $L^{2}(\Omega)$ has a normal derivative $\left(\partial_{\boldsymbol{n}} v\right)_{\mid \Upsilon}$ that belongs to $H^{-1 / 2}(\Upsilon)$. We refer to [1] for a detailed study of the fractional Sobolev spaces.

\section{EXAMPLES OF UNilATERAL CONDITIONS}

A large number of thermal, mechanical or electrical devices can be modeled by unilateral boundary conditions. We refer the reader to the book by Duvaut and Lions [17] for various examples. In fact the two examples we describe here are picked up from that reference. 


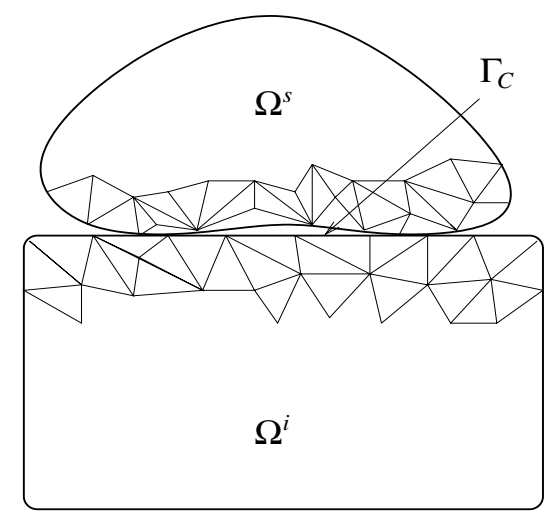

Figure 1 . Two solids are in unilateral contact along $\Gamma_{C}$. Inter-penetration is not authorized.

\subsection{Unilateral contact between two solids}

Let us consider two elastic solids occupying the domains $\Omega^{s}$ and $\Omega^{i}$ in their initial unconstrained configurations. They are assumed in contact with each other along a common part of their boundaries $\left(\partial \Omega^{s} \cap \partial \Omega^{i}\right)$. These boundaries $\partial \Omega^{s}$ and $\partial \Omega^{i}$ are supposed regular and the unit external normal is denoted by $\boldsymbol{n}$ when no confusion is feared or $\boldsymbol{n}^{i}$ and $\boldsymbol{n}^{s}$ for the corresponding bodies. Each of $\partial \Omega^{\ell}(\ell=i$ or $s$ ) is divided into three portions $\Gamma_{D}^{\ell}, \Gamma_{N}^{\ell}$ et $\Gamma_{C}^{\ell}$ (see Fig. 1). The solid $\Omega^{\ell}$ is fixed along $\Gamma_{D}^{\ell}$ and is subjected to a traction or compression force $\boldsymbol{g}^{\ell}\left(\in \boldsymbol{L}^{2}\left(\Gamma_{N}^{\ell}\right)\right)$ on $\Gamma_{N}^{\ell}$ and to a volume force $\boldsymbol{f}^{\ell}\left(\in \boldsymbol{L}^{2}\left(\Omega^{\ell}\right)\right)$, which is most often its own weight. Both solids share a common zone $\Gamma_{C}=\Gamma_{C}^{s}=\Gamma_{C}^{i}$, candidate to an effective contact.

The unilateral contact problem consists in : finding a displacement fields $\boldsymbol{u}=\left(\boldsymbol{u}^{\ell}\right)_{\ell}=\left(\boldsymbol{u}_{\mid \Omega^{i}}, \boldsymbol{u}_{\mid \Omega^{s}}\right)$ satisfying the boundary value problem

$$
\begin{aligned}
& -\operatorname{div} \sigma^{\ell}\left(\boldsymbol{u}^{\ell}\right)=\boldsymbol{f}^{\ell} \quad \text { in } \quad \Omega^{\ell}, \\
& \boldsymbol{u}^{\ell}=0 \quad \text { on } \quad \Gamma_{D}^{\ell}, \\
& \sigma^{\ell}\left(\boldsymbol{u}^{\ell}\right) \boldsymbol{n}^{\ell}=\boldsymbol{g}^{\ell} \quad \text { on } \quad \Gamma_{N}^{\ell} \text {. }
\end{aligned}
$$

The bold symbol div denotes the divergence operator of a tensor field and is defined as $\operatorname{div} \sigma=\left(\frac{\partial \sigma_{\mathbf{k r}}}{\partial \mathbf{x}_{\mathbf{r}}}\right)^{\mathbf{k}}$. The bodies are made of Hook type material whose constitutive laws are given by $\sigma^{\ell}\left(\boldsymbol{u}^{\ell}\right)=A^{\ell}(\boldsymbol{x}) \epsilon^{\ell}\left(\boldsymbol{u}^{\ell}\right)$, where $A^{\ell}(\boldsymbol{x})$ is a fourth order symmetric and elliptic tensor. Frictionless unilateral contact conditions enforced on $\Gamma_{C}$ allow to close the system

$$
\begin{gathered}
\left(\sigma^{i} \boldsymbol{n}^{i}\right) \cdot \boldsymbol{n}^{i}=\left(\sigma^{s} \boldsymbol{n}^{s}\right) \cdot \boldsymbol{n}^{s}=\sigma_{\boldsymbol{n}} \\
{[\boldsymbol{u} \cdot \boldsymbol{n}] \leq 0, \quad \sigma_{\boldsymbol{n}} \leq 0, \quad \sigma_{\boldsymbol{n}}[\boldsymbol{u} \cdot \boldsymbol{n}]=0,} \\
\sigma_{\boldsymbol{t}}^{i}=\sigma_{\boldsymbol{t}}^{s}=0 .
\end{gathered}
$$

The notation $[\boldsymbol{u} \cdot \boldsymbol{n}]=\left(\boldsymbol{u}^{i} \cdot \boldsymbol{n}^{i}+\boldsymbol{u}^{s} \cdot \boldsymbol{n}^{s}\right)$ stands for the jump of the normal displacements contact across $\Gamma_{C}$. Condition (2.4) expresses the Newton action and reaction principle, (2.6) indicates that the contact occurs without friction. Most often, the modeling of the contact condition is formulated using a gap function $\zeta$ defined on $\Gamma_{C}$, so that instead of $[\boldsymbol{u} \cdot \boldsymbol{n}] \leq 0$ and of the saturation condition $\sigma_{\boldsymbol{n}}[\boldsymbol{u} \cdot \boldsymbol{n}]=0$ we have $[\boldsymbol{u} \cdot \boldsymbol{n}] \leq \zeta$ and $\sigma_{\boldsymbol{n}}([\boldsymbol{u} \cdot \boldsymbol{n}]-\zeta)=0$ on the contact zone $\Gamma_{C}$ (see [17]). As the whole subsequent analysis can be extended straightforwardly to the case where $\zeta$ does not vanish, we choose, only for conciseness, $\zeta=0$. In what follows we will write $\sigma^{\ell}$ for $\sigma^{\ell}\left(\boldsymbol{u}^{\ell}\right)$. The analysis is limited to infinitesimal deformations (small perturbations) and therefore 
to the case of linear elasticity where the strain tensor produced by the displacement $\boldsymbol{v}$ is

$$
\epsilon(\boldsymbol{v})=\frac{1}{2}\left(\nabla \boldsymbol{v}+(\nabla \boldsymbol{v})^{T}\right)
$$

In order to derive the variational formulation of the unilateral contact problem (2.1)-(2.6) we need the spaces

$$
\boldsymbol{X}(\Omega)=\boldsymbol{H}_{0}^{1}\left(\Omega^{i}, \Gamma_{D}^{i}\right) \times \boldsymbol{H}_{0}^{1}\left(\Omega^{s}, \Gamma_{D}^{s}\right) .
$$

This space is endowed with the broken semi-norm: $\forall \boldsymbol{v} \in \boldsymbol{X}(\Omega)$,

$$
|\boldsymbol{v}|_{*, H^{1}}=\left(\left\|\epsilon\left(\boldsymbol{v}^{i}\right)\right\|_{\boldsymbol{L}^{2}\left(\Omega^{i}\right)}^{2}+\left\|\epsilon\left(\boldsymbol{v}^{s}\right)\right\|_{\boldsymbol{L}^{2}\left(\Omega^{s}\right)}^{2}\right)^{1 / 2} .
$$

which is, because of the Dirichlet condition and Korn's inequality, a norm equivalent to the broken norm $\|\cdot\|_{*, H^{1}}$. Then, the appropriate closed convex set $\boldsymbol{K}(\Omega)$ of admissible displacements is defined by

$$
\boldsymbol{K}(\Omega)=\left\{\boldsymbol{v}=\left(\boldsymbol{v}^{i}, \boldsymbol{v}^{s}\right) \in \boldsymbol{X}(\Omega), \quad([\boldsymbol{v} \cdot \boldsymbol{n}])_{\mid \Gamma_{C}} \leq 0\right\} .
$$

The weak formulation results in a variational inequality that reads as: find $\boldsymbol{u} \in \boldsymbol{K}(\Omega)$ such that

$$
a(\boldsymbol{u}, \boldsymbol{v}-\boldsymbol{u}) \geq F(\boldsymbol{v}-\boldsymbol{u}), \quad \forall \boldsymbol{v} \in \boldsymbol{K}(\Omega) .
$$

In (2.7), we set: $\forall \boldsymbol{u}, \boldsymbol{v} \in \boldsymbol{X}(\Omega)$

$$
\begin{aligned}
a(\boldsymbol{u}, \boldsymbol{v}) & =\int_{\Omega^{i}} A^{i}(\boldsymbol{x}) \epsilon\left(\boldsymbol{u}^{i}\right): \epsilon\left(\boldsymbol{v}^{i}\right) \mathrm{d} \boldsymbol{x}+\int_{\Omega^{s}} A^{s}(\boldsymbol{x}) \epsilon\left(\boldsymbol{u}^{s}\right): \epsilon\left(\boldsymbol{v}^{s}\right) \mathrm{d} \boldsymbol{x}, \\
F(\boldsymbol{v}) & =\int_{\Omega^{i}} \boldsymbol{f}^{i} \cdot \boldsymbol{v}^{i} \mathrm{~d} \boldsymbol{x}+\int_{\Gamma_{\boldsymbol{g}}^{i}} \boldsymbol{g}^{i} \cdot \boldsymbol{v}^{i} \mathrm{~d} \Gamma+\int_{\Omega^{s}} \boldsymbol{f}^{s} \cdot \boldsymbol{v}^{s} \mathrm{~d} \boldsymbol{x}+\int_{\Gamma_{\boldsymbol{g}}^{s}} \boldsymbol{g}^{s} \cdot \boldsymbol{v}^{s} \mathrm{~d} \Gamma,
\end{aligned}
$$

The existence and uniqueness of $\boldsymbol{u} \in \boldsymbol{K}(\Omega)$ which solves problem (3.5) result from Stampacchia's Theorem (see $[22,31])$.

\subsection{The Signorini-Laplace problem}

Consider the domain $\Omega$ as the union of two non-intersecting components $\Omega^{s}$ and $\Omega^{i}$. Assume that both sub-domains share a common boundary $\Gamma_{C}$. Then, denote by $\Gamma_{N}^{s}$ and $\Gamma_{N}^{i}$ the remaining parts of the boundaries of $\Omega^{s}$ and $\Omega^{i}$, respectively. We set $\Gamma_{N}=\Gamma_{N}^{s} \cup \Gamma_{N}^{i}$ and $\Gamma^{\ell}=\Gamma_{C} \cup \Gamma_{N}^{\ell}$ for $\ell=i, s$. Finally, let $\boldsymbol{n}_{C}(\boldsymbol{n}$ if there is no risk of confusion) be the unit normal to $\Gamma_{C}$ oriented from $\Omega^{i}$ toward $\Omega^{s}$.

Assume now that $g=\left(g^{s}, g^{i}\right) \in L^{2}\left(\Gamma_{N}\right)$ and $f \in L^{2}(\Omega)$. The unilateral contact problem we interested in consists in finding $p=\left(p^{i}, p^{s}\right)$, satisfying the following equations:

$$
\begin{aligned}
& p^{\ell}-\operatorname{div}\left(a^{\ell} \nabla p^{\ell}\right)=f \quad \text { in } \quad \Omega^{\ell}, \ell=i, s, \\
& \left(a^{\ell} \partial_{\boldsymbol{n}}\right) p^{\ell}=g^{\ell} \quad \text { on } \quad \Gamma_{N}^{\ell}, \ell=i, s .
\end{aligned}
$$

The coefficient $a=\left(a^{i}, a^{s}\right)$ lies in $L^{\infty}(\Omega)$. It is such that $0<a_{*} \leq a(\boldsymbol{x}) \leq a^{*}<\infty, \forall \boldsymbol{x} \in \Omega$. To complete the model, unilateral conditions should be provided along $\Gamma_{C}$. They read as

$$
\begin{aligned}
& \left(a^{i} \partial_{\boldsymbol{n}}\right) p^{i}=\left(a^{s} \partial_{\boldsymbol{n}}\right) p^{s}, \\
{[p]=p^{i}-p^{s} \geq 0, \quad } & \left(a^{i} \partial_{\boldsymbol{n}}\right) p^{i} \geq 0, \quad\left(a^{i} \partial_{\boldsymbol{n}}\right) p^{i}[p]=0 .
\end{aligned}
$$

We aim at a variational framework fitted to the problem (2.8)-(2.11). It is provided by the broken space

$$
H_{*}^{1}(\Omega)=H^{1}\left(\Omega^{i}\right) \times H^{1}\left(\Omega^{s}\right) .
$$


The broken norm is denoted by $\|\cdot\|_{*, H^{1}}$. Taking into account the unilateral contact condition on $\Gamma_{C}$ in the weak formulation is ensured by incorporating it in the closed convex cone

$$
K(\Omega)=\left\{q \in H_{*}^{1}(\Omega), \quad([q])_{\mid \Gamma_{C}}=\left(q^{i}-q^{s}\right)_{\mid \Gamma_{C}} \geq 0\right\} .
$$

The primal variational principle applied to problem (2.8)-(2.11) produces the variational inequality consisting in: finding $p \in K(\Omega)$ such that:

$$
a(p, q-p) \geq F(q-p), \quad \forall q \in K(\Omega) .
$$

In (2.12), we have set

$$
\begin{aligned}
a(p, q) & =\int_{\Omega} p q \mathrm{~d} \boldsymbol{x}+\int_{\Omega^{i}} a^{i}(\nabla p)(\nabla q) \mathrm{d} \boldsymbol{x}+\int_{\Omega^{s}} a^{s}(\nabla p)(\nabla q) \mathrm{d} \boldsymbol{x}, \\
F(v) & =\int_{\Omega} f q \mathrm{~d} \boldsymbol{x}+\langle g, q\rangle_{1 / 2, \Gamma}
\end{aligned}
$$

Applying here again Stampacchia's theorem (see [22]), we state that the variational inequality (2.12) is well posed and has only one solution in $p \in K(\Omega)$ that depends continuously on the data $(f, g)$.

Remark 2.1. In the variational formulation, the mathematical sense given to conditions (2.11) is as follows

$$
\begin{gathered}
\left\langle\left(a^{i} \partial_{\boldsymbol{n}}\right) p, \mu\right\rangle_{1 / 2, \Gamma_{C}} \geq 0, \quad \forall \mu \in H^{1 / 2}\left(\Gamma_{C}\right), \mu \geq 0, \\
\left\langle\left(a^{i} \partial_{\boldsymbol{n}}\right) p^{i},[p]\right\rangle_{1 / 2, \Gamma_{C}}=0 .
\end{gathered}
$$

Roughly, the first formula says $\left(a^{i} \partial_{\boldsymbol{n}}\right) p^{i} \geq 0$ on $\Gamma_{C}$ while the second expresses the saturation condition $\left(a^{i} \partial_{\boldsymbol{n}}\right) p^{i}[p]=0$ on $\Gamma_{C}$.

Remark 2.2. Variational inequality $(2.12)$ can be viewed as the optimality condition of a quadratic minimization problem on the closed convex $K(\Omega)$. Hence, the solution $p \in K(\Omega)$ is the one that satisfies

$$
\frac{1}{2} a(p, p)-F(p)=\min _{q \in K(\Omega)} \frac{1}{2} a(q, q)-F(q) .
$$

\subsection{About singularities}

The numerical analysis of any finite element method applied the unilateral contact problem (2.12) requires the knowledge of the regularity of the solution $p=\left(p^{i}, p^{s}\right)$. Since the work by Moussaoui and Khodja (see [36]), it is admitted that the unilateral condition may generate some singular behavior at the neighborhood of $\Gamma_{C}$. We assume $\Gamma_{C}$ is the union of regular portions connected to each other through some corners. Some singularities are generated by the angular interfaces and some others are specifically created by the contact condition. We will assume that $a^{\ell}=1$, this is the most easy case to chack out for the regularity of the Signorini solution.

Let us first look at the portions of the unilateral boundary that are straight-lines. The unilateral contact on $\Gamma_{C}$ creates some singularities caused by the points where the contact changes from binding ${ }^{6}\left(p^{s}=p^{i}\right)$ to non-binding $\left(p^{i}>p^{s}\right)$. Let $\boldsymbol{m}$ be one of those points. Considering the global pressure $p=\left(p^{i}, p^{s}\right)$ at the neighborhood of $\boldsymbol{m}$, we have the transmission conditions $\left(p^{i}, \partial_{\boldsymbol{n}} p^{i}\right)=\left(p^{s}, \partial_{\boldsymbol{n}} p^{s}\right)$ along the binding portion of $\Gamma_{C}$. As a result, $p$ is harmonic at the neighborhood of that binding boundary. Things happen as if we were involved in a Poisson equation around $\boldsymbol{m}$ and the non-binding part of $\Gamma_{C}$ acts like an insulating crack. The shape of the singularities affecting both $\left(p^{s}, p^{i}\right)$ are

$$
\begin{array}{ll}
\mathcal{S}_{k}^{i}(r, \theta)=\alpha_{k} \varphi(r) r^{k+1 / 2} \cos (k+1 / 2) \theta, & 0 \leq \theta \leq \pi, \\
\mathcal{S}_{k}^{s}(r, \theta)=\alpha_{k} \varphi(r) r^{k+1 / 2} \cos (k+1 / 2) \theta, & \pi \leq \theta \leq 2 \pi
\end{array}
$$

\footnotetext{
${ }^{6}$ We adopt here the terminology from the solid mechanics.
} 
The polar coordinates $(r, \theta)$ are used with origin $\boldsymbol{m}$ and $\varphi$ is a cut-off function around $\boldsymbol{m}$. The effective contact takes place for $\theta=\pi$ and the pressure $p$ is discontinuous at $\theta=0(2 \pi)$. Writing the unilateral conditions (2.10)-(2.11) at both sides of $\boldsymbol{m}$

$$
\mathcal{S}_{k}^{i}(r, 0)-\mathcal{S}_{k}^{s}(r, 2 \pi)>0, \quad \partial_{n} S_{k}^{i}(r, \pi)=\partial_{n} S_{k}^{s}(r, \pi) \geq 0
$$

produces $\alpha_{k}\left(1-(-1)^{k}\right)>0$ and $(-1)^{k+1} \alpha_{k} \geq 0$. This yields that $k$ is necessarily odd and $\alpha_{k}>0$. Then only the odd $k$-singularities are involved in the expansion of $p^{\ell}$, produces that

$$
p^{\ell}(r, \theta)=p_{r e g}^{\ell}(r, \theta)+\sum_{k} \mathcal{S}_{2 k+1}^{\ell}(r, \theta) .
$$

We expect therefore that $p^{\ell} \in H^{\sigma}\left(V_{\boldsymbol{m}}\right)$ for any $\sigma<\frac{5}{2}$ and $V_{\boldsymbol{m}}$ is a an open set containing $\boldsymbol{m}$. Furthermore, if the first singularities $\left(\mathcal{S}_{1}^{\ell}\right)_{\ell}$ are switched off then the next ones $\left(\mathcal{S}_{3}^{\ell}\right)_{\ell}$ command the regularity of $\left(p^{i}, p^{s}\right)$, at least at the neighborhood of $\Gamma_{C}$ and one obtains that $p^{\ell} \in H^{\sigma}\left(V_{m}\right)$ for any $\sigma<\frac{9}{2}$. In fact, the point to be fixed once for all is that the possible values of the limit Sobolev regularity index increases by jumps of length two, it equals to $5 / 2,9 / 2, \cdots$. Now, let us focus on the corners. At a vertex $\boldsymbol{m}$ with an aperture of the angle $\omega \in] 0,2 \pi\left[^{7}\right.$, three situations are possible. Either, effective contact holds along both edges of the sector, and the global solution $\left(p^{i}, p^{s}\right)$ is blind to the interface and then non singularities arise. The second situation is where effective contact takes place along one edge while on the other we have no contact. This configuration is a reminiscence to the upper case of a straight-line. The binding edge has no role to play in the shape of the singularities arising around $\boldsymbol{m}$. Only singularities like those discussed for a straight-line enter the asymptotic expansions of $\left(p^{i}, p^{s}\right)$. It is noticeable that these first two possibilities for corners do not degrade the regularity of the solution $\left(p^{i}, p^{s}\right)$, already limited by the changing of the contact conditions along a straight-line. The last case consists in the absence of contact on both sides $\left(p^{i}<p^{s}\right)$. The homogeneous normal derivative of both $\left(p^{i}, p^{s}\right)$ allows to compute the admissible singularities,

$$
\begin{aligned}
& \mathcal{S}_{k}^{i}(r, \theta)=\eta_{k}+\alpha_{k} \varphi(r) r^{\frac{k \pi}{\omega}}|\log r| \cos \frac{k \pi}{\omega} \theta, \quad 0 \leq \theta \leq \omega, \\
& \mathcal{S}_{k}^{s}(r, \theta)=\beta_{k} \varphi(r) r^{\frac{k \pi}{2 \pi-\omega}}|\log r| \cos \frac{k \pi}{2 \pi-\omega}(2 \pi-\theta) \quad \omega \leq \theta \leq 2 \pi .
\end{aligned}
$$

where $\eta_{k}>0$ is sufficiently large to ensure that $p^{i}>p^{s}$ within the support of $\varphi$ (at the neighborhood of the corner $\boldsymbol{m}$ ). Moreover, the logarithm is there for $\mathcal{S}_{k}^{i}$ only when $\frac{k \pi}{\omega}$ is an integer, idem for $\mathcal{S}_{k}^{s}$ and $\frac{k \pi}{2 \pi-\omega}$. The regularity of the first singular functions $\left(\mathcal{S}_{1}^{i}, \mathcal{S}_{1}^{s}\right)$ will fully decide of the smoothness of $\left(p^{i}, p^{s}\right)$. This predicts that $p^{i} \in H^{\sigma}\left(V_{\Gamma_{C}}\right)$ for any $\sigma<1+\frac{\pi}{\omega}$ and $p^{s} \in H^{\sigma}\left(V_{\boldsymbol{m}}\right)$ for any $\sigma<1+\frac{\pi}{2 \pi-\omega}$. Anyway, we obtain $p^{\ell} \in H^{3 / 2}\left(V_{\Gamma_{C}}\right)$. Would these singularities be switched off, there would be a jump on the regularity exponents, since $5 / 2<\sigma<$ $1+\min \left(\frac{2 \pi}{\omega}, \frac{2 \pi}{2 \pi-\omega}\right)$ and so on.

\section{The QUADRATIC MORTAR FINITE ELEMENT METHOD}

We pursue the numerical simulation of the unilateral boundary conditions by non-matching finite element grids. The main advantage is the possible generation of meshes well adapted to the local features of the domain components and to the physical parameters. As said earlier, the convergence rate of the finite element approximation depends on the regularity of the solution $p$. According the previous regularity discussion it may reasonably happen that $p$ belongs to a more regular space than $H^{3 / 2}$ or even than $H^{5 / 2}$, at least near $\Gamma_{C}$. Therefore, the approximation of the variational inequality (2.12) by affine finite elements fails to fully account for that regularity of $p$ (at least at the vicinity of $\Gamma_{C}$ ). We are indeed limited by $H^{2}$. Quadratic finite elements for the Signorini problem have been analyzed in $[4,27]$. Many factors command the accuracy of the discretization

\footnotetext{
${ }^{7} \omega$ is the angle within $\Omega^{i}$. The angle in $\Omega^{s}$ is then $(2 \pi-\omega)$.
} 
such as the smoothness of $\Gamma_{C}$, the regularity of $f$ and $g$, the way $\Gamma_{C}$ is approximated by a given mesh $\ldots$, and the unilateral singularities along $\Gamma_{C}$. Our scrutiny work is exclusively oriented toward the effect of this last point, and we do not pay attention to the other factors. They are all classical and have been considered in many works (see $[9,15])$. Assume then that the domain $\Omega$ is polygonal so that it can be exactly covered by rectilinear finite elements and that $\Gamma_{C}$ is a union of straight-lines.

Towards the construction of the discrete space, we recall some fundamental notions. Let $h=\left(h_{i}, h_{s}\right)$ be a given pair of real positive numbers that will decay towards zero. With each $\Omega^{\ell}$, we associate a regular family of triangulations $\mathcal{T}_{h}^{\ell}$, with triangular elements, denoted by $\kappa$, whose diameter does not exceed $h_{\ell}$. The unilateral boundary $\Gamma_{C}$ inherits two independent meshes $\mathcal{T}_{h}^{\ell, C}(\ell=i, s)$ from $\mathcal{T}_{h}^{\ell}$. The mesh $\mathcal{T}_{h}^{\ell, C}$ on $\Gamma_{C}$ is the set of all

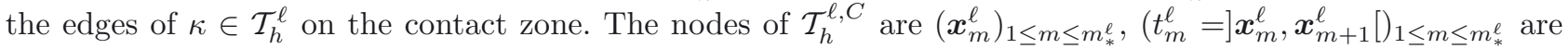
the edges and the middle nodes of $t_{m}$ is $\boldsymbol{x}_{m+1 / 2}^{\ell}$. Denote by $\mathcal{P}_{2}$ the space of the polynomials on $\kappa$ with a global degree $\leq 2$. With any $\kappa$, we associate the finite set $\Xi_{\kappa}$ of the vertices of $\kappa$, so that $\left(\kappa, \mathcal{P}_{2}, \Xi_{\kappa}\right)$ is a finite element of Lagrange type. We define $\Xi^{\ell}=\bigcup_{\kappa \in \mathcal{T}_{h}^{\ell}} \Xi_{\kappa}$. The finite element space in $\Omega^{\ell}$ is then

$$
X_{h}\left(\Omega^{\ell}\right)=\left\{q_{h}^{\ell} \in \mathscr{C}\left(\bar{\Omega}^{\ell}\right), \quad \forall \kappa \in \mathcal{T}_{h}^{\ell}, \quad\left(q_{h}^{\ell}\right)_{\mid \kappa} \in \mathcal{P}_{2}\right\}
$$

and $X_{h}(\Omega)=X_{h}\left(\Omega^{i}\right) \times X_{h}\left(\Omega^{s}\right)$. The unilateral condition (2.11) in a discrete level should be driven carefully. Comparing directly $q_{h}^{i}$ and $q_{h}^{s}$, built on different meshes, has to be avoided since it is known to create numerical troubles. It is rather recommended to realize it between $q_{h}^{i}$ and some projection of $q_{h}^{s}$ on the opposite mesh $\mathcal{T}_{h}^{i, C}$. We therefore need the traces space

$$
W_{h}^{\ell}\left(\Gamma_{C}\right)=\left\{\varphi_{h}=\left(q_{h}^{\ell}\right)_{\mid \Gamma_{C}}, \quad q_{h}^{\ell} \in X_{h}\left(\Omega^{\ell}\right)\right\}
$$

which will be the mortar space for a fixed $\ell$. Given that $\Gamma_{C}$ is a closed curve there is no need to differentiate the Lagrange Multipliers space and the mortar space as currently made (see [8]). The mortar projection $\pi^{\ell}$ is defined on $W_{h}^{\ell}\left(\Gamma_{C}\right)$ as follows. Let $\psi \in L^{2}\left(\Gamma_{C}\right)$, we have $\pi^{\ell} \psi \in W_{h}^{\ell}\left(\Gamma_{C}\right)$ and

$$
\int_{\Gamma_{C}}\left(\psi-\pi^{\ell} \psi\right) \chi_{h} \mathrm{~d} \Gamma=0 \quad \forall \chi_{h} \in W_{h}^{\ell}\left(\Gamma_{C}\right)
$$

The operator $\pi^{\ell}$ is an orthogonal projection with respect to the norm of $L^{2}\left(\Gamma_{C}\right)$ and is therefore expected to satisfy an optimal error estimate with respect to the norm of that space. It is also mandatory that it yields an optimal estimate for the norm of $H^{1 / 2}\left(\Gamma_{C}\right)$. We hence add a mild assumption on the mesh $\mathcal{T}_{h}^{\ell, C}$ introduced in [16]: two arbitrary edges $t_{m}^{\ell}$ and $t_{m^{\prime}}^{\ell}$ satisfy

$$
\frac{\left|t_{m}^{\ell}\right|}{\left|t_{m^{\prime}}^{\ell}\right|} \leq \eta \alpha^{\left|m-m^{\prime}\right|}, \quad\left(1 \leq m, m^{\prime} \leq m_{*}^{\ell}\right),
$$

where $1 \leq \alpha<9$ and $\eta$ does not depend on $h_{s} . \mathcal{T}_{h}^{\ell, C}$ is then called an (M)-mesh ${ }^{8}$. As a result, the following stability holds: $\forall \psi \in H^{1 / 2}\left(\Gamma_{C}\right)$,

$$
\left\|\pi^{\ell} \psi\right\|_{H^{1 / 2}\left(\Gamma_{C}\right)} \leq C\|\psi\|_{H^{1 / 2}\left(\Gamma_{C}\right)} .
$$

The proof may be issued from the results in $[16,39]$ and an interpolation artifice of linear operators in Hilbert spaces. Moreover, $\pi^{\ell}$ satisfies some approximation results. Let $\mu \in[0,3[$, the following approximation holds: $\forall \psi \in H^{\mu}\left(\Gamma_{C}\right)$,

$$
\left\|\psi-\pi^{\ell} \psi\right\|_{H^{-1 / 2}\left(\Gamma_{C}\right)}+h_{\ell}\left\|\psi-\pi^{\ell} \psi\right\|_{H^{1 / 2}\left(\Gamma_{C}\right)} \leq C\left(h_{\ell}\right)^{\mu+1 / 2}\|\psi\|_{H^{\mu}\left(\Gamma_{C}\right)} .
$$

We proceed with the construction of the discrete convex cone. We have then to decide which side of $\Gamma_{C}$ plays the role of the mortar, let us say $\Omega^{i}$, the same $\Gamma_{C}$ as a part of $\partial \Omega^{s}$ is hence the non-mortar. The purpose

\footnotetext{
${ }^{8}(\mathrm{M})$-meshes authorizes the adaptativity contrary to the quasi-uniform meshes.
} 


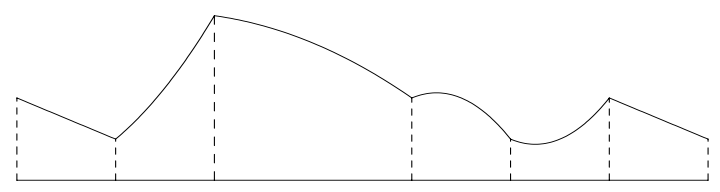

Figure 2. A test functions $\psi_{h}$. Observe that its affine at both extreme edges.

consists in enforcing the non-negativity on the values of $\left(\pi^{s}\left(q_{h}^{i}\right)-q_{h}^{s}\right)$ at the vertices of $\Gamma_{C}$ coming from the $\Omega^{s}$ side. The discrete closed convex cone is hence defined to be

$$
K_{h}(\Omega)=\left\{q_{h}=\left(q_{h}^{s}, q_{h}^{i}\right) \in X_{h}(\Omega), \quad\left[q_{h}\right]_{h}(\boldsymbol{x})=\left(\pi^{s}\left(q_{h}^{i}\right)-q_{h}^{s}\right)(\boldsymbol{x}) \geq 0, \quad \forall \boldsymbol{x} \in \Xi^{s} \cap \Gamma_{C}\right\} .
$$

The overall tools useful for the discrete variational inequality are available. The Ritz-Galerkin approximation reads as follows: find $p_{h} \in K_{h}(\Omega)$ such that

$$
a\left(p_{h}, q_{h}-p_{h}\right) \geq F\left(q_{h}-p_{h}\right), \quad \forall q_{h} \in K_{h}(\Omega) .
$$

The set $K_{h}(\Omega)$ is obviously an external approximation of $K(\Omega)$, i.e. $K_{h}(\Omega) \not \subset K(\Omega)$, the mortar discretization is of course non-conforming. Checking that the discrete problem (3.4) has only one solution $p_{h} \in K_{h}(\Omega)$ is an easy matter from Stampacchia's Theorem.

Remark 3.1. It is possible to split $\Gamma_{C}$ into more than one mortar (resp. non-mortar), each edge of $\Omega^{i}$ (resp. $\Omega^{s}$ ) may be considered as mortar (resp. non-mortar) by itself. The matching projection has to be particularized to the non mortars, whose union forms $\Gamma_{C}$ as part of $\Omega^{s}$. Each of these projections should be defined in the specific mortaring way (see $[8,41]$ ). The test functions $\psi_{h}$ involved in the definition of [8] has to be reshaped as depicted in Figure 2. Nevertheless, for the issue under examination, the theory for the mortar method may be conducted similarly. We do not therefore consider this case in details.

\section{Convergence of the Mortar method}

The analysis of the accuracy of the approximation, as said earlier, will be specifically dedicated to the effect of unilateral condition, excluding the other factors such as the regularity of the data that have been widely investigated in the literature. Henceforth, $f$ and $g$ are taken regular. Moreover, for seek of simplicity we fix the parameter $a$ to unity, that is $a^{i}=a^{s}=1$. The forthcoming results are extended to the general case provided that $a$ is smooth enough.

Relying on the discussion in Section 2.3, we will pay a particular attention to the case where the regularity exponent of the solution $p$ is either $3 / 2$ or $5 / 2$. The proofs for $\sigma=3 / 2$ can be reproduced, with a minor adaptation, when the regularity exponent $\sigma$ lies in $[1,3 / 2$ [ and those developed for $\sigma=5 / 2$ can be extended to the case where $\sigma \in] 2,5 / 2$ [ following the same lines. In the subsequent the generic constant $C$ depends on the solution $p=\left(p^{i}, p^{s}\right)$. Our main result in this paper, that we state here and prove in several steps, is the following.

Theorem 4.1. Let $p=\left(p^{i}, p^{s}\right) \in K(\Omega)$ be the solution of the variational inequality (2.12). Assume that $p^{\ell} \in$ $H^{3 / 2}\left(\Omega^{\ell}\right)$. Then we have that

$$
\left\|p-p_{h}\right\|_{*, H^{1}} \leq C\left[\left(h_{i}\right)^{1 / 2}+\left(h_{s}\right)^{1 / 2}\right] .
$$

Assume that $p^{\ell} \in H^{5 / 2}\left(\Omega^{\ell}\right)$. Then we have that

$$
\left\|p-p_{h}\right\|_{*, H^{1}} \leq C\left[\left(h_{i}\right)^{3 / 2}+\left(h_{s}\right)^{3 / 2}\right] .
$$

The constant $C$ is dependent on $\left\|p^{\ell}\right\|_{H^{\sigma}\left(\Omega^{\ell}\right)}$. 
Remark 4.2. The analysis of the intermediary case $\sigma \in] 3 / 2,2]$ requires some more work. The important fact to point at is the necessity here of a technical assumption on the effective contact region that seems to be firstly introduced in [12]. The effective contact portion of $\Gamma_{C}$ is supposed to be the union of a finite number of segments. In addition, some Sobolev and Sobolev Murray embeddings combined to finite element approximation results Lebesgue spaces $L^{r}(r>2)$ are needed in the proof.

Remark 4.3. Notice that the issue of optimal convergence rates for higher regularity that is $\sigma \in] 5 / 2,3]$ is still open. This has nothing to do with the mortaring process. Such results are also missing for the Signorini problem where only a single mesh is involved.

\subsection{Falk's Lemma in the Mortar context}

Deriving an estimate of the error $\left(p-p_{h}\right)$ with respect to the energy norm relies on a basic tool obtained from the Falk Lemma (see $[5,19])$.

Lemma 4.4. Let $p \in K(\Omega)$ be the solution of the variational inequality $(2.12)$ and $p_{h} \in K_{h}(\Omega)$ be the solution of the discrete variational inequality (3.4). Then we have that

$$
\left\|p-p_{h}\right\|_{*, H^{1}}^{2} \leq C\left\{\inf _{q_{h} \in K_{h}(\Omega)}\left(\left\|p-q_{h}\right\|_{*, H^{1}}^{2}+\left\langle\partial_{\boldsymbol{n}} p^{i},\left[q_{h}\right]\right\rangle_{1 / 2, \Gamma_{C}}\right)+\inf _{q \in K(\Omega)}\left\langle\partial_{\boldsymbol{n}} p^{i},\left[q-p_{h}\right]\right\rangle_{1 / 2, \Gamma_{C}}\right\} .
$$

Remark 4.5. The last infimum is the consistency error, it is the "variational crime" and is due to the nonconformity of the approximation.

\subsection{The best approximation error}

We pursue an optimal bound of the first infimum in the abstract Falk's estimate (4.1). The following holds.

Proposition 4.6. Assume that $p^{\ell} \in H^{\sigma}\left(\Omega^{\ell}\right)$ with $\sigma=3 / 2$ or $5 / 2$. Then we have that

$$
\inf _{q_{h} \in K_{h}(\Omega)}\left(\left\|p-q_{h}\right\|_{*, H^{1}}^{2}+\left\langle\partial_{\boldsymbol{n}} p^{i},\left[q_{h}\right]\right\rangle_{1 / 2, \Gamma_{C}}\right) \leq C\left[\left(h_{i}\right)^{\sigma-1}+\left(h_{s}\right)^{\sigma-1}\right]^{2} .
$$

Proof. Observe first that $[p]=\left(p^{i}-p^{s}\right) \in H^{\sigma-1 / 2}\left(\Gamma_{C}\right)$. Using the lifting theorem (see [1]) we may find $r \in$ $H^{\sigma}\left(\Omega^{s}\right)$ such that $r_{\mid \Gamma_{C}}=[p] \geq 0$ verifying the stability

$$
\|r\|_{H^{\sigma}\left(\Omega^{s}\right)} \leq C\left[\left\|p^{i}\right\|_{H^{\sigma}\left(\Omega^{i}\right)}+\left\|p^{s}\right\|_{H^{\sigma}\left(\Omega^{s}\right)}\right] .
$$

Setting $\left(\tilde{p}^{i}, \tilde{p}^{s}\right)=\left(p^{i}, p^{s}+r\right) \in H^{\sigma}\left(\Omega^{i}\right) \times H^{\sigma}\left(\Omega^{s}\right)$, it is clear that $[\tilde{p}]=0$ on $\Gamma_{C}$ and thus $\tilde{p} \in H^{1}\left(\Omega^{i} \cup \Omega^{s}\right)$. We are therefore in the bilateral contact context. Proceeding as in [5] (see also [8]) we are able to build $\left(\tilde{q}_{h}^{i}, \tilde{q}_{h}^{s}\right) \in X_{h}\left(\Omega^{i}\right) \times X_{h}\left(\Omega^{s}\right)$ satisfying the mortar matching that is $\left[\tilde{q}_{h}\right]_{h}=0$. Moreover, thanks to (3.2) there holds that

$$
\left\|p-\tilde{q}_{h}\right\|_{*, H^{1}} \leq C\left[\left(h_{i}\right)^{\sigma-1}+\left(h_{s}\right)^{\sigma-1}\right] .
$$

Now, let $r_{h} \in X_{h}\left(\Omega^{s}\right)$ be the Lagrange interpolant of $r$ and define $q_{h}$ as follows

$$
q_{h}=\left(q_{h}^{i}, q_{h}^{s}\right)=\left(\tilde{q}_{h}^{i}, \tilde{q}_{h}^{s}-r_{h}\right) .
$$

We check easily that, for all $\boldsymbol{x} \in \Xi^{s} \cap \Gamma_{C}$, we have

$$
\left[q_{h}\right]_{h}(\boldsymbol{x})=r_{h}(\boldsymbol{x})=r(\boldsymbol{x})=[p](\boldsymbol{x}) \geq 0 .
$$

As a result, we have that $q_{h} \in K_{h}(\Omega)$ and the following bound holds

$$
\left\|p-q_{h}\right\|_{*, H^{1}} \leq\left\|p-\tilde{q}_{h}\right\|_{*, H^{1}}+\left\|r-r_{h}\right\|_{H^{1}\left(\Omega^{s}\right)} \leq C\left\|p-\tilde{q}_{h}\right\|_{*, H^{1}}+C\left(h_{s}\right)^{\sigma-1}\|r\|_{H^{\sigma}\left(\Omega^{s}\right)}
$$


Using (4.4) together with (4.3) yields

$$
\left\|p-\tilde{q}_{h}\right\|_{*, H^{1}} \leq C\left[\left(h_{i}\right)^{\sigma-1}+\left(h_{s}\right)^{\sigma-1}\right] .
$$

The first part of the infimum (4.2) is bounded in an optimal way. There remains to estimate $\left\langle\partial_{\boldsymbol{n}} p^{i},\left[q_{h}\right]\right\rangle_{1 / 2, \Gamma_{C}}$ which is pretty longer. Furthermore, different technicalities are required according to the regularity exponent $\sigma$. We choose to provide the proofs into two separate lemmas that come just after. Once these are stated, the proof of the proposition will be completed.

Lemma 4.7. Assume that $p^{\ell} \in H^{3 / 2}\left(\Omega^{\ell}\right)$ and $q_{h}$ given as in (4.5). Then we have that

$$
\left\langle\partial_{\boldsymbol{n}} p^{i},\left[q_{h}\right]\right\rangle_{1 / 2, \Gamma_{C}} \leq C\left[\left(h_{i}\right)^{1 / 2}+\left(h_{s}\right)^{1 / 2}\right]^{2} .
$$

Proof. From the saturation $\left(\partial_{n} p^{i}\right)[p]=0$ on $\Gamma_{C}$, we derive that

$$
\begin{aligned}
\left\langle\partial_{\boldsymbol{n}} p^{i},\left[q_{h}\right]\right\rangle_{1 / 2, \Gamma_{C}} & =\left\langle\partial_{\boldsymbol{n}} p^{i},\left[\tilde{q}_{h}\right]\right\rangle_{1 / 2, \Gamma_{C}}+\left\langle\partial_{\boldsymbol{n}} p^{i}, r_{h}\right\rangle_{1 / 2, \Gamma_{C}} \\
& =\left\langle\partial_{\boldsymbol{n}} p^{i},\left[\tilde{q}_{h}\right]\right\rangle_{1 / 2, \Gamma_{C}}+\left\langle\partial_{\boldsymbol{n}} p^{i},\left(r_{h}-r\right)\right\rangle_{1 / 2, \Gamma_{C}} .
\end{aligned}
$$

The first term is handled in a standard way (see $[5,8]$ ). There remains to bound the second one. That $p^{i} \in$ $H^{3 / 2}\left(\Omega^{i}\right)$ with $(-\Delta) p^{i} \in L^{2}\left(\Omega^{i}\right)$ results in $\left(\partial_{\boldsymbol{n}} p^{i}\right) \in L^{2}\left(\Gamma_{C}\right)$ (see [1]). We can then write that

$$
\begin{aligned}
\left\langle\partial_{\boldsymbol{n}} p^{i},\left(r_{h}-r\right)\right\rangle_{1 / 2, \Gamma_{C}} & =\int_{\Gamma_{C}}\left(\partial_{\boldsymbol{n}} p^{i}\right)\left(r_{h}-r\right) \mathrm{d} \Gamma \leq\left\|\partial_{\boldsymbol{n}} p^{i}\right\|_{L^{2}\left(\Gamma_{C}\right)}\left\|r-r_{h}\right\|_{L^{2}\left(\Gamma_{C}\right)} \\
& \leq C\left(h_{s}\right)\left\|\partial_{\boldsymbol{n}} p^{i}\right\|_{L^{2}\left(\Gamma_{C}\right)}\|r\|_{H^{1}\left(\Gamma_{C}\right)} \leq C\left(h_{s}\right)\left\|p^{i}\right\|_{H^{3 / 2}\left(\Omega^{i}\right)}\|r\|_{H^{3 / 2}\left(\Omega^{s}\right)} .
\end{aligned}
$$

Using (4.3) with $\sigma=3 / 2$, completes the proof.

Lemma 4.8. Assume that $p^{\ell} \in H^{5 / 2}\left(\Omega^{\ell}\right)$ and $q_{h}$ given as in (4.5). Then we have that

$$
\left\langle\partial_{\boldsymbol{n}} p^{i},\left[q_{h}\right]\right\rangle_{1 / 2, \Gamma_{C}} \leq C\left[\left(h_{i}\right)^{3 / 2}+\left(h_{s}\right)^{3 / 2}\right]^{2} .
$$

Proof. The proof is a slightly more complicated than in the previous case. Again, the point is to bound the second term of (4.6). Notice that, due to the regularity of $p^{\ell}$, we obtain

$$
\begin{aligned}
\int_{\Gamma_{C}}\left(\partial_{\boldsymbol{n}} p^{i}\right)\left(r_{h}-r\right) \mathrm{d} \Gamma & =\int_{\Gamma_{C}}\left(\partial_{\boldsymbol{n}} p^{i}\right)\left(r_{h}-r\right) \mathrm{d} \Gamma \\
& =\sum_{i=1}^{m^{*}} \int_{t_{m}}\left(\partial_{\boldsymbol{n}} p^{i}\right)\left(r-r_{h}\right) \mathrm{d} \Gamma \leq \sum_{i=1}^{m^{*}}\left\|\partial_{\boldsymbol{n}} p^{i}\right\|_{L^{2}\left(t_{m}\right)}\left\|r-r_{h}\right\|_{L^{2}\left(t_{m}\right)} .
\end{aligned}
$$

Some indices in the sum may be removed. Are kept there only the indices $m \in \tilde{M}$ for which $\left(\partial_{n} p^{i}\right)_{\mid \Gamma_{C}} \subset$ $H^{1}\left(\Gamma_{C}\right) \subset \mathscr{C}\left(\Gamma_{C}\right)$ vanishes at least once in $t_{m}$. Otherwise if $\left(\partial_{\boldsymbol{n}} p^{i}\right)_{\mid t_{m}}>0$ then $r_{\mid t_{m}}=[p]_{\mid t_{m}}=0$. This gives $\left(r_{h}\right)_{\mid t_{m}}=0$ and the associated integral is zero. Then, the inequality reduces to

$$
\int_{\Gamma_{C}}\left(\partial_{\boldsymbol{n}} p^{i}\right)\left(r_{h}-r\right) \mathrm{d} \Gamma \leq C \sum_{m \in \tilde{M}}\left\|\partial_{\boldsymbol{n}} p^{i}\right\|_{L^{2}\left(t_{m}\right)}\left(h_{s}\right)^{2}|r|_{H^{2}\left(t_{m}\right)} .
$$

Applying now Lemma 8.1 of [4] to $\partial_{\boldsymbol{n}} p^{i} \in H^{1}\left(\Gamma_{C}\right)$, we obtain

$$
\begin{aligned}
\int_{\Gamma_{C}}\left(\partial_{\boldsymbol{n}} p^{i}\right)\left(r_{h}-r\right) \mathrm{d} \Gamma & \leq \sum_{m \in \tilde{M}} C\left(h_{i}\right)\left|\partial_{\boldsymbol{n}} p^{i}\right|_{H^{1}\left(t_{m}\right)}\left(h_{s}\right)^{2}|r|_{H^{2}\left(t_{m}\right)} \\
& \leq C\left(h_{i}\right)\left(h_{s}\right)^{2}\left(\sum_{m \in \tilde{M}}\left|\partial_{\boldsymbol{n}} p^{i}\right|_{H^{1}\left(t_{m}\right)}^{2}\right)^{1 / 2}\left(\sum_{m \in \tilde{M}}|r|_{H^{2}\left(t_{m}\right)}^{2}\right)^{1 / 2} \\
& \leq C\left(h_{i}\right)\left(h_{s}\right)^{2}\left|\partial_{\boldsymbol{n}} p^{i}\right|_{H^{1}\left(\Gamma_{C}\right)}|r|_{H^{2}\left(\Gamma_{C}\right)} .
\end{aligned}
$$

The proof is achieved due to the estimate (4.3) with $\sigma=5 / 2$. 


\subsection{The consistency error}

Now, we are left with the consistency error. The analysis of this error requires some sharp technicalities.

Lemma 4.9. Assume that $p^{\ell} \in H^{3 / 2}\left(\Omega^{\ell}\right)$. Then we have that

$$
\inf _{q \in K(\Omega)}\left\langle\partial_{\boldsymbol{n}} p^{i},\left[q-p_{h}\right]\right\rangle_{1 / 2, \Gamma_{C}} \leq C\left[\left(h_{s}\right)^{1 / 2}\left\|p-p_{h}\right\|_{*, H^{1}}+\left(h_{s}\right)\right] .
$$

Proof. The proof takes four (arborescent) steps, though not that difficult. Given that $\left(\partial_{\boldsymbol{n}} p^{i}\right) \in L^{2}\left(\Gamma_{C}\right)$, taking $q=p$ yields that

$$
\left\langle\partial_{\boldsymbol{n}} p^{i},\left[p-p_{h}\right]\right\rangle_{1 / 2, \Gamma_{C}}=\int_{\Gamma_{C}}\left(\partial_{\boldsymbol{n}} p^{i}\right)\left[p-p_{h}\right] \mathrm{d} \Gamma .
$$

Plugging $\left[p-p_{h}\right]_{h}$ results in

$$
\left\langle\partial_{\boldsymbol{n}} p^{i},\left[p-p_{h}\right]\right\rangle_{1 / 2, \Gamma_{C}}=\int_{\Gamma_{C}}\left(\partial_{\boldsymbol{n}} p^{i}\right)\left[p-p_{h}\right]_{h} \mathrm{~d} \Gamma-\int_{\Gamma_{C}}\left(\partial_{\boldsymbol{n}} p^{i}\right)\left(\left(I-\pi^{s}\right)\left(p^{i}-p_{h}^{i}\right)\right) \mathrm{d} \Gamma .
$$

Both integrals involved in there are handled separately. We start by the second integral because it is shorter.

(i) By the Cauchy-Schwarz inequality we have that

$$
\int_{\Gamma_{C}}\left(\partial_{\boldsymbol{n}} p^{i}\right)\left(\left(I-\pi^{s}\right)\left(p^{i}-p_{h}^{i}\right)\right) \mathrm{d} \Gamma \leq\left\|\partial_{\boldsymbol{n}} p^{i}\right\|_{L^{2}\left(\Gamma_{C}\right)}\left\|\left(I-\pi^{s}\right)\left(p^{i}-p_{h}^{i}\right)\right\|_{L^{2}\left(\Gamma_{C}\right)} .
$$

Owing to (3.3), we obtain that

$$
\int_{\Gamma_{C}}\left(\partial_{\boldsymbol{n}} p^{i}\right)\left(\left(I-\pi^{s}\right)\left(p^{i}-p_{h}^{i}\right)\right) \mathrm{d} \Gamma \leq C\left(h_{s}\right)^{1 / 2}\left\|p^{i}-p_{h}^{i}\right\|_{H^{1 / 2}\left(\Gamma_{C}\right)} \leq C\left(h_{s}\right)^{1 / 2}\left\|p-p_{h}\right\|_{*, H^{1}} .
$$

(ii) Now, we turn to estimating the first integral in the bound of (4.7). Let $\chi_{h}$ be the orthogonal projection of $\left(\partial_{\boldsymbol{n}} p^{i}\right)$ on the piecewise constant functions built on the mesh $\mathcal{T}_{h}^{s, C}$. Actually $\left(\left(\chi_{h}\right)_{\mid t_{m}}\right)_{m}$ are obtained as the average values of $\left(\partial_{\boldsymbol{n}} p^{i}\right)$ on $\left(t_{m}\right)_{m}$ and are then non-negative. Additionally we have the following bound (see [2]),

$$
\left\|\left(\partial_{\boldsymbol{n}} p^{i}\right)-\chi_{h}\right\|_{H^{-1 / 2}\left(\Gamma_{C}\right)} \leq C\left(h_{s}\right)^{1 / 2}\left\|\partial_{\boldsymbol{n}} p^{i}\right\|_{L^{2}\left(\Gamma_{C}\right)} \leq C\left(h_{s}\right)^{1 / 2}\left\|p^{i}\right\|_{H^{3 / 2}\left(\Omega^{i}\right)} .
$$

The derivation of the desired bound is based on the following decomposition

$$
\int_{\Gamma_{C}}\left(\partial_{\boldsymbol{n}} p^{i}\right)\left[p-p_{h}\right]_{h} \mathrm{~d} \Gamma=\int_{\Gamma_{C}}\left(\left(\partial_{\boldsymbol{n}} p^{i}\right)-\chi_{h}\right)\left[p-p_{h}\right]_{h} \mathrm{~d} \Gamma+\int_{\Gamma_{C}} \chi_{h}\left[p-p_{h}\right]_{h} \mathrm{~d} \Gamma .
$$

Processing in the same lines as above we come up with the estimation

$$
\int_{\Gamma_{C}}\left(\left(\partial_{\boldsymbol{n}} p^{i}\right)-\chi_{h}\right)\left[p-p_{h}\right]_{h} \mathrm{~d} \Gamma \leq C\left(h_{s}\right)^{1 / 2}\left\|p-p_{h}\right\|_{*, H^{1}} .
$$

In the other side, using the Simpson formula and given that $\chi_{h} \geq 0$, and $\left[p_{h}\right]_{h} \geq 0$, it is possible to get rid of $p_{h}$ to obtain that

$$
\int_{\Gamma_{C}} \chi_{h}\left[p-p_{h}\right]_{h} \mathrm{~d} \Gamma \leq \int_{\Gamma_{C}} \chi_{h}[p]_{h} \mathrm{~d} \Gamma
$$

or again that

$$
\int_{\Gamma_{C}} \chi_{h}\left[p-p_{h}\right]_{h} \mathrm{~d} \Gamma \leq \int_{\Gamma_{C}} \chi_{h}[p] \mathrm{d} \Gamma+\int_{\Gamma_{C}} \chi_{h}\left(p^{i}-\pi^{s}\left(p^{i}\right)\right) \mathrm{d} \Gamma .
$$

Let us, here, look at each bound independently. 
(iii) Using Cauchy-Schwarz' inequality we have that

$$
\begin{aligned}
\int_{\Gamma_{C}} \chi_{h}\left(\left(p^{i}-\pi^{s}\left(p^{i}\right)\right) \mathrm{d} \Gamma\right. & \leq\left\|\chi_{h}\right\|_{L^{2}\left(\Gamma_{C}\right)}\left\|p^{i}-\pi^{s}\left(p^{i}\right)\right\|_{L^{2}\left(\Gamma_{C}\right)} \\
& \leq C\left\|\partial_{\boldsymbol{n}} p^{i}\right\|_{L^{2}\left(\Gamma_{C}\right)}\left(h_{s}\right)\left\|p^{i}\right\|_{H^{1}\left(\Gamma_{C}\right)} \leq C h_{s} .
\end{aligned}
$$

(iv) The bound of the remaining term is processed as follows. We need $\eta_{h}$ the orthogonal projection of $[p]$ on the piecewise constant functions built on the mesh $\mathcal{T}_{h}^{s, C}$. The unilateral conditions and that $\chi_{h}$ is the orthogonal projection of $\left(\partial_{\boldsymbol{n}} p^{i}\right)$ allows to write

$$
\begin{aligned}
\int_{\Gamma_{C}} \chi_{h}[p] \mathrm{d} \Gamma & =\int_{\Gamma_{C}}\left(\chi_{h}-\left(\partial_{\boldsymbol{n}} p^{i}\right)\right)[p] \mathrm{d} \Gamma \\
& =\int_{\Gamma_{C}}\left(\chi_{h}-\left(\partial_{\boldsymbol{n}} p^{i}\right)\right)\left([p]-\eta_{h}\right) \mathrm{d} \Gamma=-\int_{\Gamma_{C}}\left(\partial_{\boldsymbol{n}} p^{i}\right)\left([p]-\eta_{h}\right) \mathrm{d} \Gamma .
\end{aligned}
$$

Another use of the Cauchy-Schwarz' inequality yields that

$$
\int_{\Gamma_{C}} \chi_{h}[p] \mathrm{d} \Gamma \leq\left\|\partial_{\boldsymbol{n}} p^{i}\right\|_{L^{2}\left(\Gamma_{C}\right)}\left\|[p]-\eta_{h}\right\|_{L^{2}\left(\Gamma_{C}\right)} \leq C\left\|\left(\partial_{\boldsymbol{n}} p^{i}\right)\right\|_{L^{2}\left(\Gamma_{C}\right)}\left(h_{i}\right)\|[p]\|_{H^{1}\left(\Gamma_{C}\right)} \leq C\left(h_{i}\right) .
$$

The proof is achieved by aggregating the bounds of $(i),(i i i)$ and $(i v)$.

Before handling the case where $p^{\ell} \in H^{5 / 2}\left(\Omega^{\ell}\right)$, we need a preparatory lemma. Let $t$ be a segment with length $h=|t|$. The following holds.

Lemma 4.10. For any $\left.\alpha \in] \frac{3}{2}, 2\right]$, there exists a constant $C>0$ so that: $\forall \psi \in H^{\alpha}(t), \forall a \in t$,

$$
\left\|\psi(x)-\left(\psi(a)+\psi^{\prime}(a)(x-a)\right)\right\|_{L^{2}(t)} \leq C h^{\alpha}|\psi|_{H^{\alpha}(t)} .
$$

The constant $C$ does not depend on a.

Proof. Consider for a while the reference segment $\hat{t}=(0,1)$. Then the Sobolev space $H^{\alpha}(\hat{t})$ is continuously embedded in the space $\mathscr{C}^{1}(\hat{t})$ (see [1]). Let $\hat{\psi} \in H^{\alpha}(\hat{t})$. Set $\hat{\lambda}(\hat{x})=\hat{\psi}(\hat{x})-(c+\mathrm{d} \hat{x})$. It is straightforward that $\hat{\lambda} \in H^{\alpha}(\hat{t})$ and we have

$$
\sup _{\hat{x} \in \hat{t}}\left|\hat{\lambda}(\hat{x})-\left(\hat{\lambda}(\hat{a})+\hat{\lambda}^{\prime}(\hat{a})(\hat{x}-\hat{a})\right)\right| \leq \hat{c}\|\hat{\lambda}\|_{H^{\alpha}(\hat{t})},
$$

or again that

$$
\sup _{\hat{x} \in \hat{t}}\left|\hat{\psi}(\hat{x})-\left(\hat{\psi}(\hat{a})+\hat{\psi}^{\prime}(\hat{a})(\hat{x}-\hat{a})\right)\right| \leq \hat{c}\|\hat{\psi}-(c \hat{x}+d)\|_{H^{\alpha}(\hat{t})} .
$$

Since $(c, d)$ are arbitrary, calling for the Bramble-Hilbert Theorem we have

$$
\sup _{\hat{x} \in \hat{t}}\left|\hat{\psi}(\hat{x})-\left(\hat{\psi}(\hat{a})+\hat{\psi}^{\prime}(\hat{a})(\hat{x}-a)\right)\right| \leq \hat{c} \inf _{c, d \in \mathbb{R}}\|\hat{\psi}-(c+\mathrm{d} \hat{x})\|_{H^{\alpha}(\hat{t})} \leq \hat{c}|\hat{\psi}|_{H^{\alpha}(\hat{t})} .
$$

Then, we derive that

$$
\left\|\hat{\psi}(\hat{x})-\left(\hat{\psi}(\hat{a})+\hat{\psi}^{\prime}(\hat{a})(\hat{x}-\hat{a})\right)\right\|_{L^{2}(\hat{t})} \leq\left\|\hat{\psi}(\hat{x})-\left(\hat{\psi}(\hat{a})+\hat{\psi}^{\prime}(\hat{a})(\hat{x}-\hat{a})\right)\right\|_{L^{\infty}(\hat{t})} \leq \hat{c}|\hat{\psi}|_{H^{\alpha}(\hat{t})} .
$$

A scaling transformation $\hat{x} \mapsto x$ and $\psi(x)=\hat{\psi}(\hat{x})$ provide the lemma with $C=\hat{c}$.

Lemma 4.11. Assume that $p^{\ell} \in H^{5 / 2}\left(\Omega^{\ell}\right)$. Then we have that

$$
\inf _{q \in K(\Omega)}\left\langle\partial_{\boldsymbol{n}} p^{i},\left[q-p_{h}\right]\right\rangle_{1 / 2, \Gamma_{C}} \leq C\left[\left(h_{i}\right)^{3 / 2}\left\|p-p_{h}\right\|_{*, H^{1}}+\left(h_{s}\right)^{3}\right] .
$$


Proof. The structure of the proof is similar to the previous lemma, though some modifications are necessary.

(i) Setting $\psi_{h}=\pi^{s}\left(\partial_{\boldsymbol{n}} p^{i}\right)$, the duality allows to write that

$$
\begin{aligned}
\int_{\Gamma_{C}}\left(\partial_{\boldsymbol{n}} p^{i}\right)\left(\left(I-\pi^{s}\right)\left(p^{i}-p_{h}^{i}\right)\right) \mathrm{d} \Gamma & =\int_{\Gamma_{C}}\left(\left(\partial_{\boldsymbol{n}} p^{i}\right)-\psi_{h}\right)\left(\left(I-\pi^{s}\right)\left(p^{i}-p_{h}^{i}\right)\right) \mathrm{d} \Gamma \\
& \leq\left\|\left(\partial_{\boldsymbol{n}} p^{i}\right)-\psi_{h}\right\|_{H^{-1 / 2}\left(\Gamma_{C}\right)}\left\|\left(I-\pi^{s}\right)\left(p^{i}-p_{h}^{i}\right)\right\|_{H^{1 / 2}\left(\Gamma_{C}\right)} .
\end{aligned}
$$

Owing to (3.2) and (3.3), we obtain that

$$
\int_{\Gamma_{C}}\left(\partial_{\boldsymbol{n}} p^{i}\right)\left(\left(I-\pi^{s}\right)\left(p^{i}-p_{h}^{i}\right)\right) \mathrm{d} \Gamma \leq C\left(h_{s}\right)^{3 / 2}\left\|p^{i}-p_{h}^{i}\right\|_{H^{1 / 2}\left(\Gamma_{C}\right)} \leq C\left(h_{s}\right)^{3 / 2}\left\|p-p_{h}\right\|_{*, H^{1}}
$$

(ii) The estimate on $\left(\left(\partial_{n} p^{i}\right)-\chi_{h}\right)$ becomes

$$
\left\|\left(\partial_{\boldsymbol{n}} p^{i}\right)-\chi_{h}\right\|_{H^{-1 / 2}\left(\Gamma_{C}\right)} \leq C\left(h_{s}\right)^{3 / 2}\left\|p^{i}\right\|_{H^{5 / 2}\left(\Omega^{i}\right)} .
$$

(iii) We have that $\left(\psi_{h}\right.$ is defined in $\left.(i)\right)$

$$
\begin{aligned}
\int_{\Gamma_{C}} \chi_{h}\left(\left(p^{i}-\pi^{s}\left(p^{i}\right)\right) \mathrm{d} \Gamma\right. & =\int_{\Gamma_{C}}\left(\chi_{h}-\left(\partial_{\boldsymbol{n}} p^{i}\right)\right)\left(p^{i}-\pi^{s}\left(p^{i}\right)\right) \mathrm{d} \Gamma+\int_{\Gamma_{C}}\left(\partial_{\boldsymbol{n}} p^{i}\right)\left(p^{i}-\pi^{s}\left(p^{i}\right)\right) \mathrm{d} \Gamma \\
& =\int_{\Gamma_{C}}\left(\chi_{h}-\left(\partial_{\boldsymbol{n}} p^{i}\right)\right)\left(p^{i}-\pi^{s}\left(p^{i}\right)\right) \mathrm{d} \Gamma+\int_{\Gamma_{C}}\left(\left(\partial_{\boldsymbol{n}} p^{i}\right)-\psi_{h}\right)\left(p^{i}-\pi^{s}\left(p^{i}\right)\right) \mathrm{d} \Gamma .
\end{aligned}
$$

Using Cauchy-Schwarz' inequality yields that

$$
\begin{aligned}
\int_{\Gamma_{C}} \chi_{h}\left(\left(p^{i}-\pi^{s}\left(p^{i}\right)\right) \mathrm{d} \Gamma\right. & \leq\left(\left\|\chi_{h}-\left(\partial_{\boldsymbol{n}} p^{i}\right)\right\|_{L^{2}\left(\Gamma_{C}\right)}+\left\|\left(\partial_{\boldsymbol{n}} p^{i}\right)-\psi_{h}\right\|_{L^{2}\left(\Gamma_{C}\right)}\right)\left\|p^{i}-\pi^{s}\left(p^{i}\right)\right\|_{L^{2}\left(\Gamma_{C}\right)} \\
& \leq C\left(h_{s}\right)\left\|\left(\partial_{\boldsymbol{n}} p^{i}\right)\right\|_{H^{1}\left(\Gamma_{C}\right)}\left(h_{s}\right)^{2}\left\|p^{i}\right\|_{H^{2}\left(\Gamma_{C}\right)} \leq C\left(h_{s}\right)^{3} .
\end{aligned}
$$

(iv) The unilateral conditions allow to write

$$
\int_{\Gamma_{C}} \chi_{h}[p] \mathrm{d} \Gamma=\int_{\Gamma_{C}}\left(\chi_{h}-\left(\partial_{\boldsymbol{n}} p^{i}\right)\right)[p] \mathrm{d} \Gamma \leq \sum_{i=1}^{m_{*}^{s}}\left\|\chi_{h}-\left(\partial_{\boldsymbol{n}} p^{i}\right)\right\|_{L^{2}\left(t_{m}\right)}\|[p]\|_{L^{2}\left(t_{m}\right)} .
$$

Arguing like in the proof of Lemma 4.8, some indices in the sum are canceled. Remains there only the indices $m \in \tilde{M}$ for which $[p]$ vanishes at least once inside $t_{m}$. Otherwise if $[p]>0$ then $\left(\partial_{\boldsymbol{n}} p\right)_{\mid t_{m}}=0$ and so does $\left(\chi_{h}\right)_{\mid t_{m}}$. This results in

$$
\int_{\Gamma_{C}} \chi_{h}[p] \mathrm{d} \Gamma=\int_{\Gamma_{C}}\left(\chi_{h}-\left(\partial_{\boldsymbol{n}} p^{i}\right)\right)[p] \mathrm{d} \Gamma \leq C\left(h_{s}\right) \sum_{i \in \tilde{M}}\left|\partial_{\boldsymbol{n}} p^{i}\right|_{H^{1}\left(t_{m}\right)}\|[p]\|_{L^{2}\left(t_{m}\right)} .
$$

Now, let us have a close look at $[p] \in \mathscr{C}^{1}\left(\Gamma_{C}\right)$ restricted to $t_{m}$ with $m \in \tilde{M}$. It vanishes at least once inside $t_{m}$, say at $a$. Given that $[p] \geq 0$, then $a$ realizes the minimum value of $[p]$. As a result, we obtain that $[p]^{\prime}(a)=0$. Next, applying Lemma 4.10 to $[p]_{\mid t_{m}}$ with $\alpha=2$, it comes out

$$
\begin{aligned}
\int_{\Gamma_{C}} \chi_{h}[p] \mathrm{d} \Gamma & \leq C\left(h_{s}\right) \sum_{i \in \tilde{M}}\left|\left(\partial_{\boldsymbol{n}} p^{i}\right)\right|_{H^{1}\left(t_{m}\right)}\|[p]\|_{L^{2}\left(t_{m}\right)} \\
& \leq C\left(h_{s}\right)^{3} \sum_{i \in \tilde{M}}\left|\left(\partial_{\boldsymbol{n}} p^{i}\right)\right|_{H^{1}\left(t_{m}\right)}|[p]|_{H^{2}\left(t_{m}\right)} \leq C\left(h_{s}\right)^{3}\left|\partial_{\boldsymbol{n}} p^{i}\right|_{H^{1}\left(\Gamma_{C}\right)}|[p]|_{H^{2}\left(\Gamma_{C}\right)} .
\end{aligned}
$$

The proof is completed after putting together the bounds of $(i),($ iii $)$ and $(i v)$. 


\subsection{Global estimations}

We are now well equipped to provide the expected optimal estimate on the error $\left(p-p_{h}\right)$ generated by the mortar quadratic finite element approximation.

Proof of Theorem 4.1. Here $\sigma$ may equal $3 / 2$ or $5 / 2$. Assembling the estimate by Proposition 4.6 and Lemma 4.9, for $\sigma=3 / 2$, or Lemma 4.11, for $\sigma=5 / 2$, produces

$$
\left\|p-p_{h}\right\|_{*, H^{1}}^{2} \leq C\left(\left[\left(h_{i}\right)^{\sigma-1}+\left(h_{s}\right)^{\sigma-1}\right]^{2}+\left(h_{i}\right)^{\sigma-1}\left\|p-p_{h}\right\|_{*, H^{1}}\right) .
$$

The proof is complete by Young's inequality.

\section{Numerical Tests}

We describe some indicative and informative numerical experiences for the Signorini-Laplace problem. The particular aim is to quantify the mortar capacity to preserve the accuracy of quadratic finite elements and to illustrate the agreement between the convergence rate observed in the computations and the one predicted by the theory. Computations for two examples are carried out by means of the code freefem++ developed by F. Hecht and his coworkers (see [23]). We recall that the computational reliability and robustness of the mortar method in the mechanical unilateral contact has already been proved in many work (see [33] and the references therein)

In both examples, the domains are rectangular, $\Omega^{s}=[-1,1] \times[-1,0]$ and $\Omega^{i}=[-1,1] \times[0,1]$. The hypothetic contact zone $\Gamma_{C}$ is the common edge $[-1,1] \times\{0\}$, Along the left (vertical) edges $\{1\} \times[-1,0]$ and $\{1\} \times[0,1]$, Neumann conditions are prescribed and along the remaining four edges we enforce Dirichlet boundary conditions. The exact solution for the first example, represented in Figure 3, is given by

$$
\begin{aligned}
& p^{i}\left(x_{1}, x_{2}\right)=x_{1} x_{2}+\left[2 \max \left(x_{1}, 0\right)-\min \left(x_{1}, 0\right) \sin \left(2 \pi x_{2}\right)\right] \sin \left(3 x_{1}\right), \\
& p^{s}\left(x_{1}, x_{2}\right)=x_{1} x_{2}+\left[-2 \max \left(x_{1}, 0\right)-\min \left(x_{1}, 0\right) \sin \left(2 \pi x_{2}\right)\right] \sin \left(3 x_{1}\right) .
\end{aligned}
$$

Notice that the unilateral contact conditions are rather expressed as follows

$$
[p] \geq 0, \quad \partial_{\boldsymbol{n}} p^{i} \geq \xi, \quad\left(\partial_{\boldsymbol{n}} p-\xi\right)[p]=0 \quad \text { on } \quad \Gamma_{C} .
$$

The gap function is $\xi\left(x_{1}\right)=-x_{1}$. The foregoing analysis remains of course valid as it is and provides the same convergence rates.

Once the discrete finite element objects are constructed, the unilateral inequality is handled as a minimization problem as formulated in (2.15). The unilateral contact conditions on the degrees of freedom located on $\Gamma_{C}$ are written under inequality constraints. The obtained optimization problem can therefore be solved iteratively by the interior point strategy, also called the barrier method (see [10]).

Now, to assess the mortar method, combined to the quadratic finite elements, we compare the results it provides to those obtained by two other procedures. The first one is related to the simple interpolation process, used on the same incompatible meshes, for the unilateral conditions. This means that the contact is expressed in a discrete level without practicing the mortar projection $\pi^{s}$. It is therefore changed to

$$
\left[q_{h}\right]_{h}(\boldsymbol{x})=\left(q_{h}^{i}-q_{h}^{s}\right)(\boldsymbol{x}) \geq 0, \quad \forall \boldsymbol{x} \in \Xi^{s} \cap \Gamma_{C} .
$$

The second approach, the mortar method is compared to, consists in considering conforming meshes with almost the same size as those used in the mortaring process. Consequently, no special projection is needed to glue the discretizations at the contact region. By the way, the mortar projection is nothing else than the identity.

Before discussing the convergence curves, let us figure out the smoothness of $p=\left(p^{i}, p^{\ell}\right) \cdot p^{i}$ and $p^{\ell}$ contain, each, a singularity at the origin $\boldsymbol{m}=(0,0)$. Indeed, $\boldsymbol{m}$ splits $\Gamma_{C}$ into an effective contact zone $\left(x_{1} \leq 0\right)$ and 

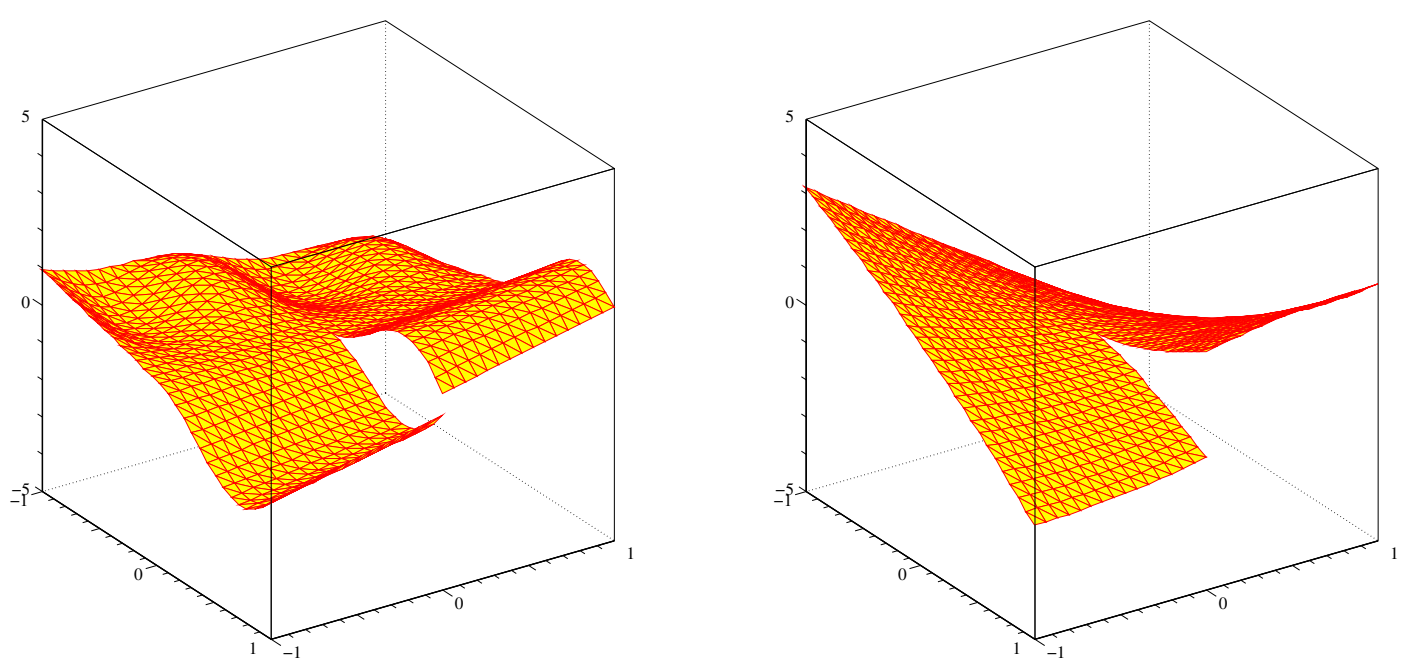

Figure 3. The mortar finite element Signorini solutions.

a non-contact $\left(x_{1} \geq 0\right)$ region. The singular effect remains limited to $\Gamma_{C}$ and is not spread in the interior of the domains. Our aim is to focus on what happen along $\Gamma_{C}$ while avoiding contamination by any other inaccuracy factors. The Sobolev regularity of both functions $p^{i}$ and $p^{\ell}$ is therefore limited to $(5 / 2)_{-}{ }^{9}$. According to Theorem 4.1, the convergence rate, with respect to the broken $H^{1}$-norm, should be close to $3 / 2$. In fact, we expect that limitation to be effective when the singular point $\boldsymbol{m}$ lies within an edge of a triangle. Otherwise, if it coincides with a vertex of some triangles, the computations may show some super-convergence result.

In all the simulations, the meshes are triangular and locally structured. We start by running computations when the singular point $\boldsymbol{m}$ is located at the middle of a triangular edge. The $H^{1}, L^{2}$ and $L^{\infty}$-convergence curves, for the conforming, the non-conforming-mortar and the non-conforming-interpolation methods are provided in Figure 4, in logarithmic scales. Obtaining the convergence rates of each method passes by the evaluation of the slopes of the error curves. Let us focus for a while on the $H^{1}$-convergence for which we dispose of theoretical estimates at least for the conforming and non-conforming mortar approximations. The linear regressions of the $H^{1}$-errors produce slopes that are close to 1.82 and 1.85 , respectively. This is slightly better than the value predicted by the analysis which is equal to 1.5. In addition, mortaring the matching enjoys an accuracy level close to that given by conforming grids. Oppositely, the slope 1.19, for the approximation based on the crude interpolation for non-matching grids, suggests that it behaves like a linear and not as a quadratic method. Actually, the results appear satisfactory for meshes of moderate sizes. Things start to worsen for fine meshes where the behavior of the interpolation matching suffers from some weakness and confirms the well known grievances about this way to proceed. A glance to the right-bottom panel in Figure 4 allows similar conclusions for the convergence rates with respect to the $L^{\infty}$-norm. The $L^{2}$-convergence curves show that the conforming and the mortar methods have similar behaviors, and the convergence rate is not that far from 2.5. In fact, after a careful examination of those curves, we found out that the slopes are slightly sagged down when the mesh size gets smaller. The evaluation of the slope of the first half of the curves result in the values 2.41 for the conforming discretization and 2.53 for the mortar method. The explanation of the sag (for the complete curves) is that the error inherent to the optimization solver grows up with the size of the discrete problem. It may then pollute the finite element error, the global accuracy of the approximation is hence affected and the convergence rate is slowed down.

\footnotetext{
${ }^{9}$ This means that $p^{\ell} \in H^{\sigma}\left(\Omega^{\ell}\right)$ for all $\sigma<5 / 2$.
} 

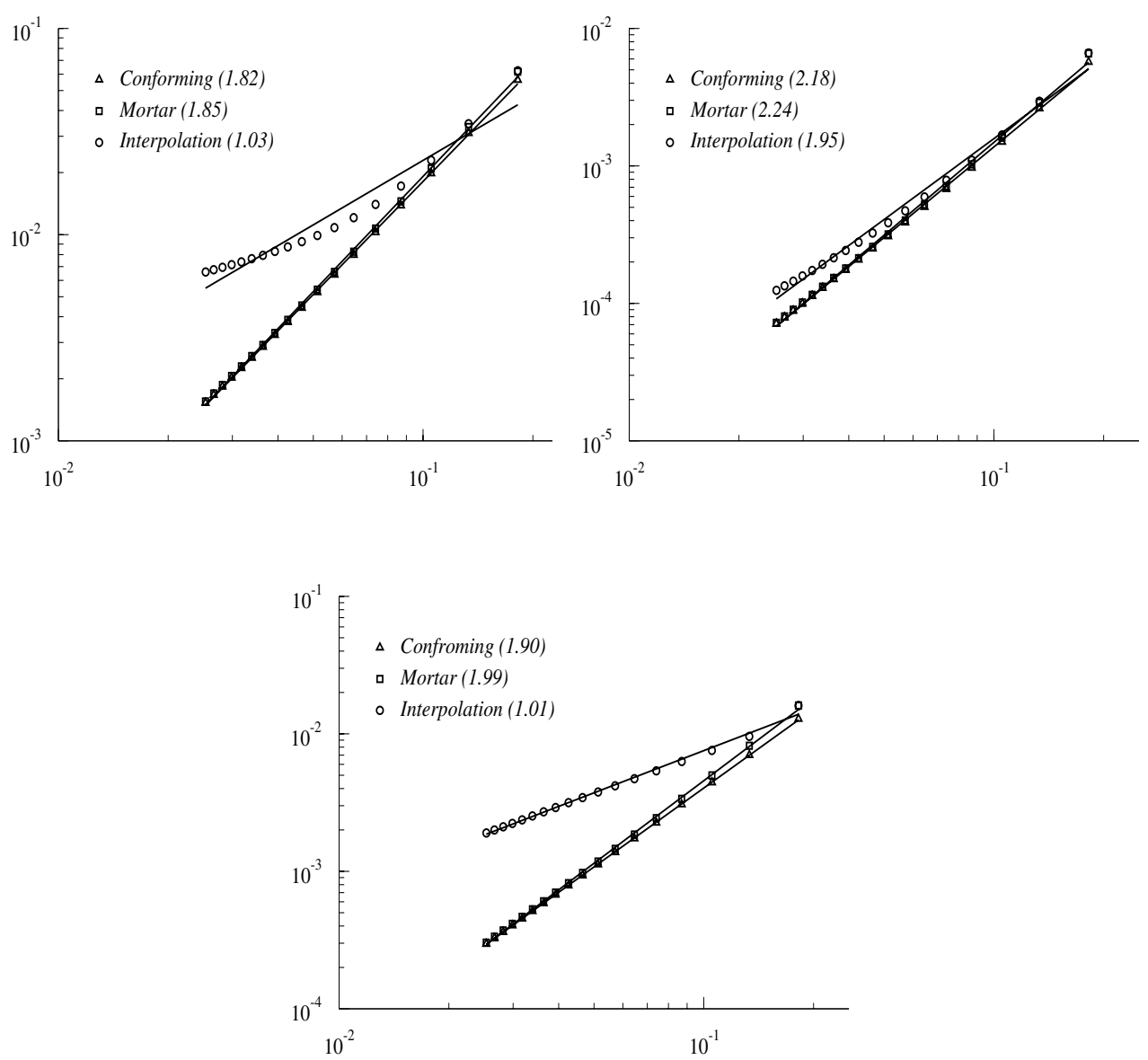

Figure 4. $H^{1}$ - (top), $L^{2}$ - (left-bottom) and $L^{\infty}$ - (right-bottom) convergence curves. The slopes of the linear regressions are provided between parenthesis in the legend.

If the singularity support is located at a vertex of some triangles then the quadratic finite element approximation may not see the full singular behavior of the solution. It remains partially blind to the jump of the second derivative of the solution with respect to $x_{1}$ at the singular point. Super-convergence may hence show up for both conforming and mortar approximations. The convergence rates with respect to the $H^{1}$-norm are almost equal to 2 , and are close to 3 for the $L^{2}$-norm. The difficulties with the interpolation matching for non-conforming grids to enjoy similar behavior are substantially aggravated. It has to be avoided, especially for methods with order higher than one. This is already widely known for the bilateral contact models. Curves in Figure 5 confirm it for the unilateral contact problems.

To close with the first example, let us draw the attention of practitioners to the way the mortar matching should be handled and implemented. The computed solution turns out to be sensitive to the accuracy of the quadrature formula used to evaluate the integrals concerning the matching formula, which is strongly impacted on the construction of the mortar projection. We resume both calculations related to the 'normal' and 'super'convergence rates. In Figure 6, are depicted the $H^{1}$-convergence curves when the mortar integrals are exactly computed and when an integration Gauss formula is used with two Gauss nodes within each edge. The plot where super-convergence is expected displays a clear degradation of the accuracy, especially for fine grids, caused by 

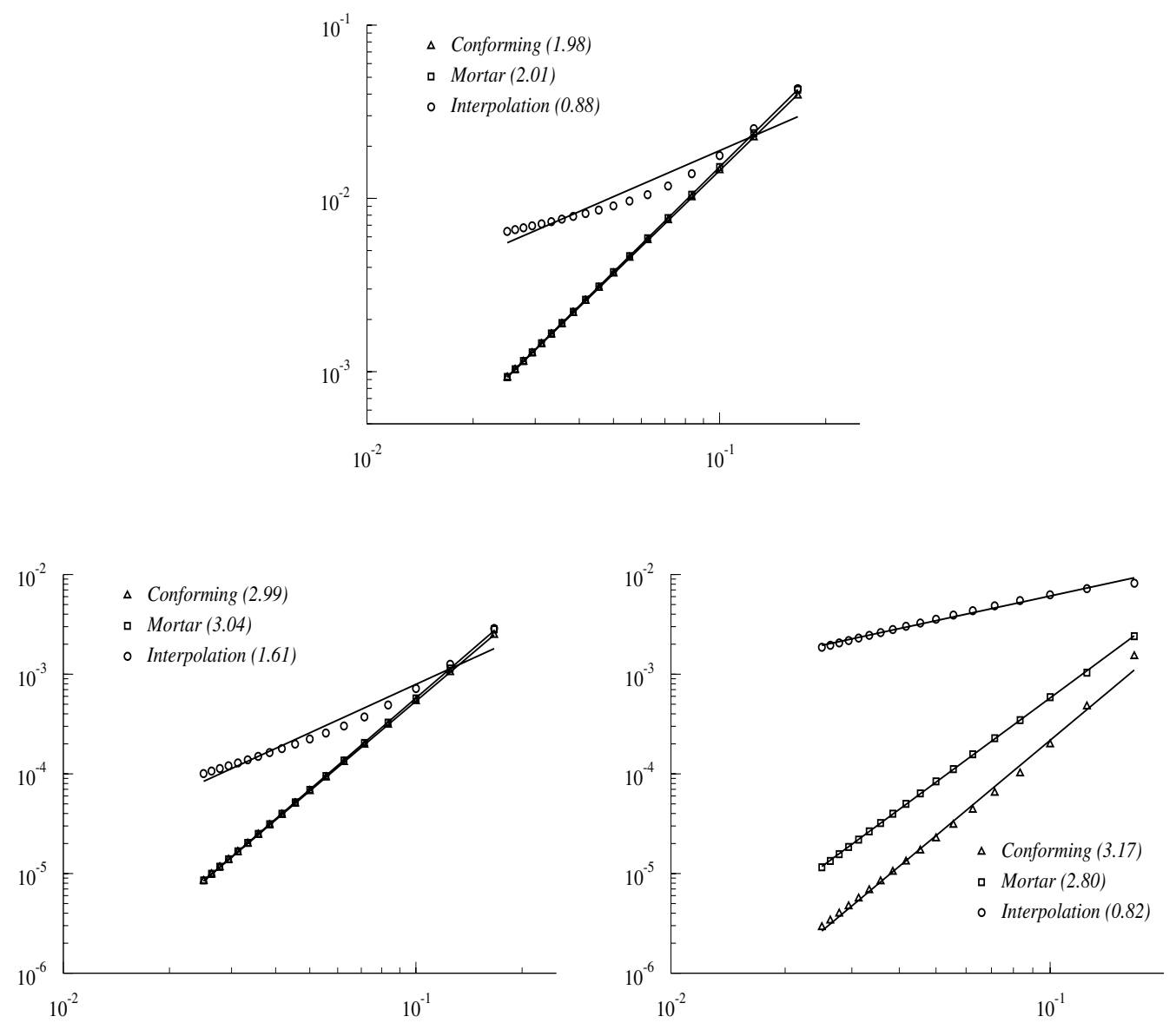

FigURE 5. Super convergence phenomenon for some particular meshes.

the numerical integration in the mortar matching. To learn more about this issue, we recommend the interesting references $[13,18]$.

The second test deals with a solution affected by the natural singularity born at the point splitting $\Gamma_{C}$ into an activated contact and non-contact zones. The exact solution is defined to be

$$
\begin{aligned}
& p^{i}\left(x_{1}, x_{2}\right)=x_{1} x_{2}+x \sqrt{r+x}-y \sqrt{r-x}, \\
& p^{s}\left(x_{1}, x_{2}\right)=x_{1} x_{2}-x \sqrt{r+x}-y \sqrt{r-x},
\end{aligned}
$$

where $r=\sqrt{x^{2}+y^{2}}$. It is depicted in the right panel of Figure 3. The non-smooth part of the solution looks like the first singularity $r^{3 / 2} \cos (3 \theta / 2)$ switched on by the unilateral contact at the vicinity of the separation point $\boldsymbol{m}$ located here again at the origin. We refer to the Section 2.3 the discussion about the singularities. The solution $p^{\ell}$ belongs to $H^{\sigma}\left(\Omega^{\ell}\right)$ for any $\sigma<\frac{5}{2}$. This indicates why the convergence rate of the quadratic finite elements should be close to $h^{3 / 2}$ with respect to the energy norm. This is confirmed by the first diagram of Figure 7, for the mortar and conforming approximations. The interpolation matching on non-conforming provides substantially less accurate results. Notice that, unlike the first example, the circular shape of the singularity contained in the solution affects the accuracy not only at the contact edge $\Gamma_{C}$ but also in the interior 

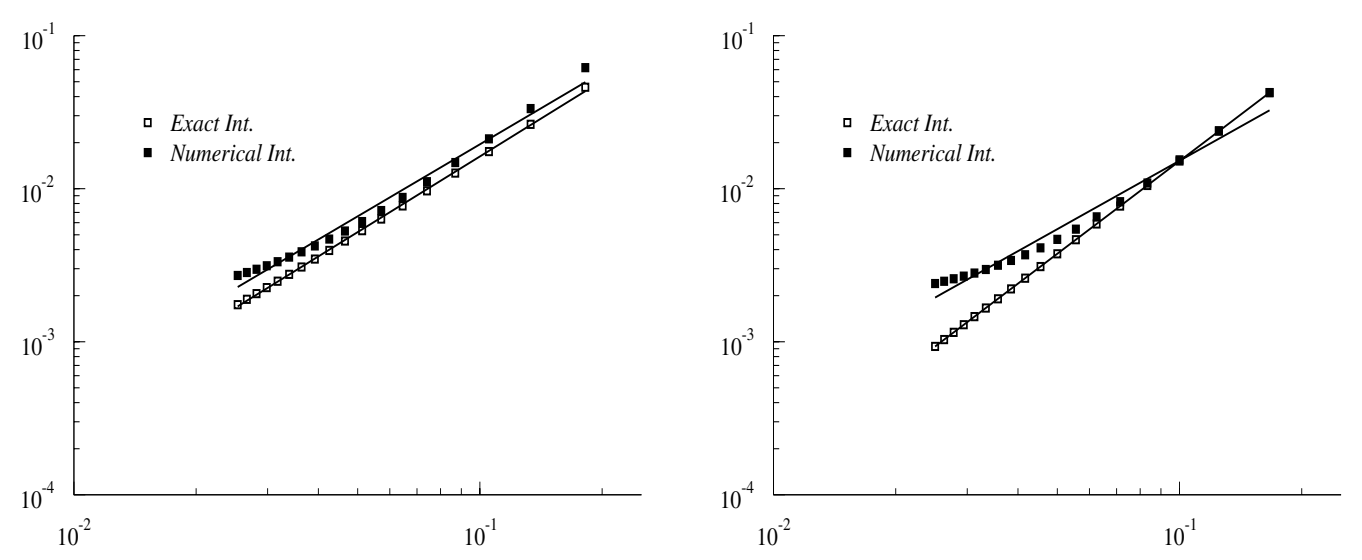

FiguRE 6 . Effect of the numerical integration on the $H^{1}$-converge. The curves to the right are related to the super-convergent case.
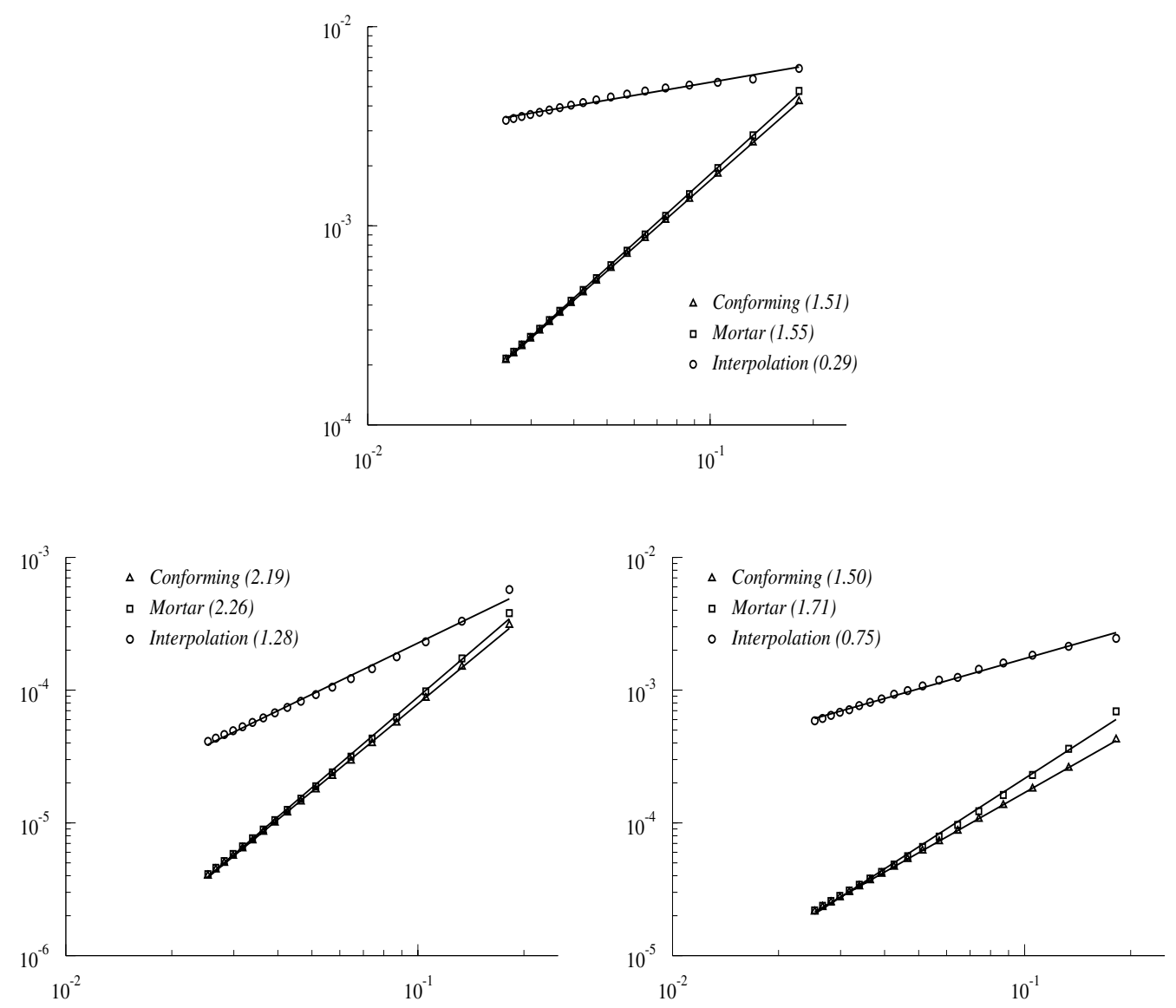

FIGURE 7. Convergence curves for the unilateral singular solution. 

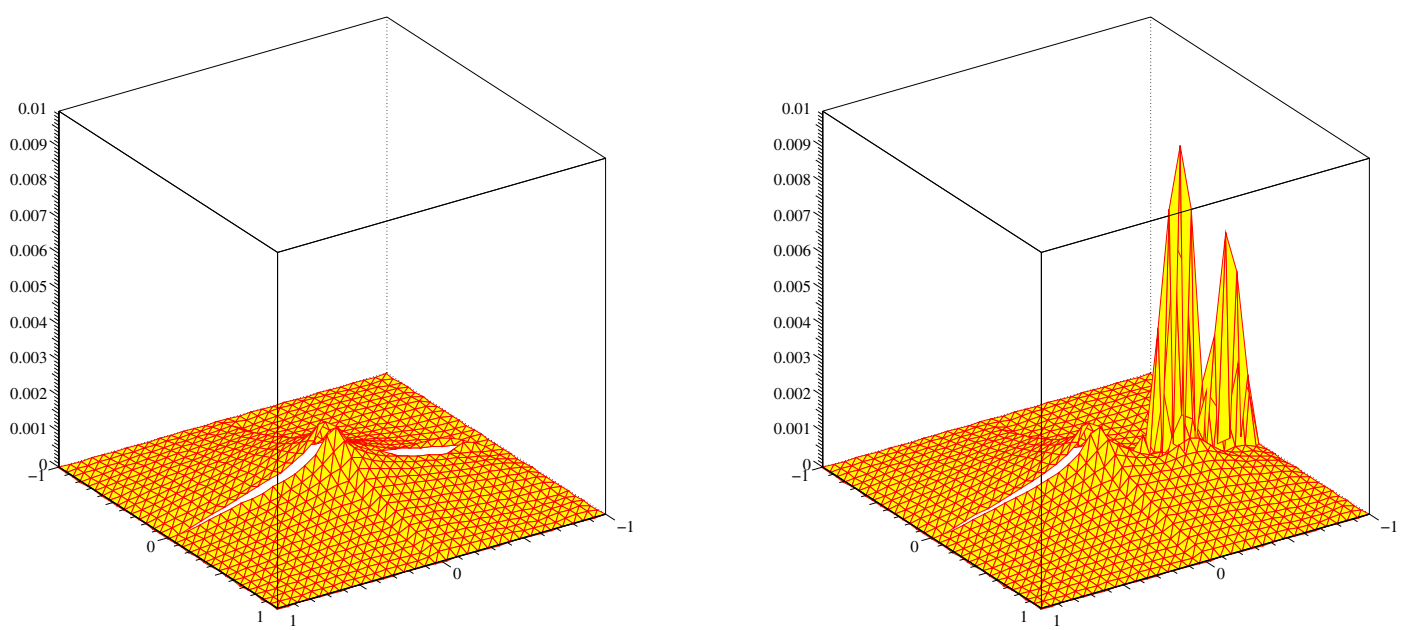

FIGURE 8. Error function for the mortar matching (left) where the singularity seems to be the main contributor to the error. The error for the interpolation matching (right) shows that high part of the error is located at the vicinity of the effective contact line, and is necessarily due to the weakness of the point-wise matching.

of the domains $\Omega^{i}$ and $\Omega^{s}$. No super-convergence phenomenon is therefore expected and the numerical results confirms this fact. There is no particular speeding of the convergence when the singular point coincides with the vertex of some triangles. Convergence curves for the $L^{2}$ and $L^{\infty}$ norms are also represented in Figure 7 . The trends observed in the first example are confirmed. In the unilateral Signorini contact, the mortar matching does not lower the convergence speed of the quadratic finite element approximation realized on non-conforming grids. In the contrary, the point-wise or the interpolation matching has to be thrown away in such a context as it yields substantially less accurate computed solutions. The distribution of the errors due to each approximation are plotted in Figure 8. The shape of these errors illustrates that, for the mortar matching, the singularity is the main cause of the limitation in the accuracy while in the interpolation matching, the way the matching is realized seems to be the principle contributor to the lost of the accuracy.

\section{Conclusion}

The numerical analysis of the quadratic finite element method with non-matching grids when applied to unilateral contact equations is achieved here. We use the mortar approach to drive the communication between different (incompatible) meshes. This mortar concept has been successfully extended to the variational inequalities in [5] for linear finite elements and employed in many computations; we refer for instance to $[3,20,26,32,37,38,43]$. The convergence rates established in this paper for the quadratic finite elements are similar to those already stated in $[4,27,28]$ when matching grids are employed. We provide some computational examples to illustrate and support the analysis. Let us mention before ending that the mortar quadratic finite element method has already been used for the computational simulation of the unilateral contact in the elasticity (see $[14,21,30,37]$ ).

Acknowledgements. The authors are indebted to anonymous reviewers whose remarks and comments substantially improves the readability of the paper. Merci to them.

\section{REFERENCES}

[1] R.A. Adams, Sobolev Spaces. Academic Press (1975).

[2] A.K. Aziz and I. Babuška, The mathematical foundations of the finite element method with applications to partial differential equations. Academic Press, New York (1972). 
[3] L. Baillet and T. Sassi, Mixed finite element formulation in large deformation frictional contact problem. European J. Comput. Mech. 14 (2005) 287-304.

[4] Z. Belhachmi and F. Ben Belgacem, Quadratic finite element for Signorini problem. Math. Comput. 72 (2003) 83-104.

[5] F. Ben Belgacem, P. Hild and P. Laborde, Extension of the mortar finite element method to a variational inequality modeling unilateral contact. Math. Models Methods Appl. Sci. 9 (1999) 287-303.

[6] F. Ben Belgacem, Y. Renard and L. Slimane, A Mixed Formulation for the Signorini Problem in nearly Incompressible Elasticity. Appl. Numer. Math. 54 (2005) 1-22.

[7] F. Ben Belgacem and Y. Renard, Hybrid finite element methods for the Signorini problem. Math. Comput. 72 (2003) $1117-1145$.

[8] C. Bernardi, Y. Maday and A.T. Patera, A New Nonconforming Approach to Domain Decomposition: The Mortar Element Method, Collège de France seminar, edited by H. Brezis, J.-L. Lions. Pitman (1994) 13-51.

[9] S.C. Brenner and L.R. Scott, Mathematical Theory of Finite Element Methods. Texts Appl. Math. Springer Verlag, New-York 15 (1994).

[10] J.-F. Bonnans, J. Ch. Gilbert, C. Lemaréchal and C.A. Sagastizábal, Numerical optimization: Theoretical and practical aspects. Universitext (Second revised ed. translation of 1997 French ed.). Springer-Verlag, Berlin (2006).

[11] F. Brezzi and M. Fortin, Mixed and Hybrid Finite Element Methods, Springer Series Comput. Math., vol. 15. Springer Verlag, New York (1991).

[12] F. Brezzi, W.W. Hager and P.A. Raviart, Error estimates for the finite element solution of variational inequalities. Numer. Math. 28 (1977) 431-443.

[13] L. Cazabeau, Y. Maday and C. Lacour, Numerical quadratures and mortar methods. In Computational Sciences for the 21-st Century, edited by Bristeau et al., Wiley and Sons (1997) 119-128.

[14] A. Chernov, M. Maischak and E.P. Stephan, $h p$-mortar boundary element method for two-body contact problems with friction. Math. Methods Appl. Sci. 31 (2008) 2029-2054.

[15] P.-G. Ciarlet, The Finite Element Method for Elliptic Problems. North Holland (1978).

[16] M. Crouzeix and V. Thomée, The Stability in $L^{p}$ and $W^{1, p}$ of the $L^{2}$-Projection on Finite Element Function Spaces. Mathods Comput. 48 (1987) 521-532.

[17] G. Duvaut and J.-P. Lions, Les inéquations en mécanique et en physique. Dunod, Paris (1972).

[18] S. Faletta, The Approximate Integration in the Mortar Method Constraint. Domain Decomposition Methods in Science and Engineering XVI. Lect. Notes Comput. Sci. Eng. Part III, 55 (2007) 555-563.

[19] R.S. Falk, Error Estimates for the Approximation of a Class of Variational Inequalities. Math. Comput. 28 963-971 (1974).

[20] K.A. Fischer and P. Wriggers, Frictionless 2D contact formulations for finite deformations based on the mortar method. Comput. Mech. 36 (2005) 226-244.

[21] B. Flemisch, M.A. Puso and B.I. Wohlmuth, A new dual mortar method for curved interfaces: 2D elasticity. Internat. J. Numer. Methods Eng. 63 (2005) 813-832.

[22] J. Haslinger, I. Hlaváček and J. Nečas, Numerical Methods for Unilateral Problems in Solid Mechanics, in Handbook of Numerical Analysis, Volume IV, Part 2, edited by P.G. Ciarlet and J.L. Lions. North Holland (1996).

[23] F. Hecht, Freefem ++ . Third Edition, Version 3.11-1 http://www.freefem.org/ff++.

[24] P. Hild and Y. Renard, An improved a priori error analysis for finite element approximations of Signorini's problem. SIAM J. Numer. Analys. to appear (2012).

[25] P. Hild, Problèmes de contact unilatéral et maillages éléments finis incompatibles. Thèse de l'Université Paul Sabatier, Toulouse 3 (1998).

[26] P. Hild, Numerical implementation of two nonconforming finite element methods for unilateral contact. Comput. Methods Appl. Mech. Eng. 184 (2000) 99-123.

[27] P. Hild, P. Laborde. Quadratic finite element methods for unilateral contact problems. Appl. Numer. Math. 41 (2002) $401-421$.

[28] D. Hua, L. Wang. A mixed finite element method for the unilateral contact problem in elasticity. Sci. China Ser. A 49 (2006) 513-524.

[29] S. Hüeber, B.I. Wohlmuth. An optimal a priori error estimate for nonlinear multibody contact problems. SIAM J. Numer. Anal. 43 (2005) 156-173.

[30] S. Hüeber, M. Mair and B.I. Wohlmuth, A priori error estimates and an inexact primal-dual active set strategy for linear and quadratic finite elements applied to multibody contact problems. Appl. Numer. Math. 54 (2005) 555-576.

[31] N. Kikuchi and J.T. Oden, Contact Problems in Elasticity: A Study of Variational Inequalities and Finite Element Methods. SIAM (1988).

[32] T.Y. Kim, J.E. Dolbow and T.A. Laursen, A Mortared Finite Element Method for Frictional Contact on Arbitrary Surfaces. Comput. Mech. 39 (2007) 223-235.

[33] T.A. Laursen, M.A. Puso and J. Sandersc, Mortar contact formulations for deformable-deformable contact: Past contributions and new extensions for enriched and embedded interface formulations. Comput. Methods Appl. Mech. Eng. 205-208 (2012) $3-15$.

[34] T.A. Laursen and B. Yang, New Developments in Surface-to-Surface Discretization Strategies for Analysis of Interface Mechanics. Computational Plasticity. Comput. Methods Appl. Sci. 7 (2010) 67-86.

[35] M.-X. Li, Q. Lin and S.-H. Zhang, Superconvergence of finite element method for the Signorini problem. J. Comput. Appl. Math. 222 (2008) 284-292.

[36] M. Moussaoui and K. Khodja, Régularité des solutions d'un problème mêlé Dirichlet-Signorini dans un domaine polygonal plan. Commun. Part. Differ. Equ. 17 (1992) 805-826. 
[37] M.A. Puso, T.A. Laursen and J. Solberg, A segment-to-segment mortar contact method for quadratic elements and large deformations. Comput. Meth. Appl. Mech. and Eng. 197 (2008) 555-566.

[38] M.A. Puso and T.A. Laursen, A Mortar Segment-to-Segment Contact Method for Large Deformation Solid Mechanics. Comput. Methods Appl. Mech. Eng. 193 (2004) 601-629.

[39] P. Seshaiyer and M. Suri, Uniform $h-p$ Convergence Results for the Mortar Finite Element Method. Math. Comput. 69 521-546 (2000).

[40] L. Slimane, Méthodes mixtes et traitement du verrouillage numérique pour la résolution des inéquations variationnelles. Thèse l'Institut National des Sciences Appliquées de Toulouse (2001).

[41] B.I. Wohlmuth, A Mortar Finite Element Method Using Dual Spaces for the Lagrange Multiplier. SIAM J. Numer. Anal. 38 (2001) 989-1012,.

[42] B. Wohlmuth, R. Krause. Monotone multigrid methods on nonmatching grids for nonlinear multibody contact problems. SIAM J. Sci. Comput. 25 (2003) 324-347.

[43] B. Yang, T.A. Laursen, X. Meng. Two dimensional mortar contact methods for large deformation frictional sliding. Internat. J. Numer. Methods Eng. 62 (2005) 1183-1225.

[44] Z.-H. Zhong, Finite Element Procedures for Contact-Impact Problems. Oxford University Press (1993). 